Pacific

Journal of

Mathematics

THE CYLINDRICAL CONTACT HOMOLOGY OF UNIVERSALLY TIGHT SUTURED CONTACT SOLID TORI

Roman GOLOVKO

Volume $274 \quad$ No. 1

March 2015 


\title{
THE CYLINDRICAL CONTACT HOMOLOGY OF UNIVERSALLY TIGHT SUTURED CONTACT SOLID TORI
}

\author{
ROMAN GOLOVKO
}

\begin{abstract}
We calculate the sutured version of cylindrical contact homology of a sutured contact solid torus $\left(S^{1} \times D^{2}, \Gamma, \xi\right)$, where $\Gamma$ consists of $2 n$ parallel sutures of arbitrary slope and $\xi$ is a universally tight contact structure. In particular, we show that it is nonzero. This computation is one of the first computations of the sutured version of cylindrical contact homology and does not follow from computations in the closed case.
\end{abstract}

\section{Introduction}

The cylindrical contact homology of a (closed) contact manifold was introduced by Eliashberg and Hofer and is the simplest version of the symplectic field theory of Eliashberg, Givental and Hofer [Eliashberg et al. 2000]. It is the homology of a differential graded module whose differential counts genus zero holomorphic curves in the symplectization with one positive puncture and one negative puncture.

In the early 1980s, Gabai [1983] developed the theory of sutured manifolds, which became a powerful tool in studying 3-manifolds with boundary. It turns out that there is a way to generalize cylindrical contact homology to sutured manifolds. This is possible by imposing a certain convexity condition on the contact form. This construction is described in the paper of Colin, Ghiggini, Honda and Hutchings [Colin et al. 2011] and will be summarized in Section 2.

In this paper, we construct a sutured contact solid torus with $2 n$ parallel sutures of slope $-k / l$ using the gluing method of [Colin et al. 2011], and calculate the sutured cylindrical contact homology of it. Here $n \in \mathbb{N},(k, l)=1$ and $k>l>0$. In order to define the slope, we choose an oriented identification $\partial\left(S^{1} \times D^{2}\right) \simeq T^{2}=(\mathbb{R} / \mathbb{Z})^{2}$ as follows: $\operatorname{map}\{p t\} \times \partial D^{2}$ (the meridian) to $(1,0)$ (slope is 0$)$ and $S^{1} \times\{p t\}$ (a longitude) to $(0,1)$.

This calculation, together with the calculation of the sutured cylindrical contact homology of the sutured contact solid torus with $2 n$ parallel longitudinal sutures, where $n \geq 2$, that has been done in [Golovko 2011], finishes the calculation of the cylindrical contact homology of $\left(S^{1} \times D^{2}, \Gamma, \xi\right)$, where $\Gamma$ consists of $2 n$ parallel

MSC2010: primary 53D42; secondary 57M50, 53D10.

Keywords: sutured manifolds, contact homology. 
sutures of arbitrary slope, $\xi$ is a universally tight contact structure and such that if one cuts along the meridian disk, the sutures on the disk are $\partial$-parallel. In particular, this gives a complete calculation of the cylindrical contact homology of $\left(S^{1} \times D^{2}, \Gamma, \xi\right)$, where $\Gamma$ consists of 2 parallel sutures of arbitrary slope and $\xi$ is a universally tight contact structure (observe that in this situation there are only two isomorphic (but not isotopic) universally tight contact structures; see [Honda 2002, Section 2]). These are not all the universally tight contact structures on the solid torus, but all of them can be obtained from the \#Г=2 case by successively applying the folding operation.

Our goal is to prove the following theorem:

Theorem 1.1. Let $\left(S^{1} \times D^{2}, \Gamma\right)$ be a sutured manifold, where $\Gamma$ is a set of $2 n$ parallel closed curves of slope $-k / l$, where $(k, l)=1, k>l>0$ and $n \in \mathbb{N}$. Then there is a contact form $\alpha$ which makes $\left(S^{1} \times D^{2}, \Gamma, \alpha\right)$ a sutured contact manifold with a universally tight contact structure $\xi=\operatorname{ker} \alpha, H C^{\mathrm{cyl}}\left(S^{1} \times D^{2}, \Gamma, \alpha\right)$ is defined, is independent of the contact form $\alpha$ for $\xi=\operatorname{ker} \alpha$ and the almost complex structure $J$ and

$$
H C^{\mathrm{cyl}, h}\left(S^{1} \times D^{2}, \Gamma, \xi\right) \simeq \begin{cases}\mathbb{Q} & \text { for } k \nmid h>0, \\ \mathbb{Q}^{n-1} & \text { for } k \mid h>0, \\ 0, & \text { otherwise. }\end{cases}
$$

Here $h$ corresponds to the homological grading.

\section{Background}

The goal of this section is to review definitions of sutured contact manifold and the relative version of cylindrical contact homology. This section can be considered as a summary of [Colin et al. 2011].

2A. Review of sutured contact manifolds. In this section, we recall some definitions and describe some constructions from [Colin et al. 2011]. We first start with the notion of a Liouville manifold.

Definition 2.1. A Liouville manifold (often also called a Liouville domain) is a pair $(W, \beta)$ consisting of a compact, oriented $2 n$-dimensional manifold $W$ with boundary and a 1-form $\beta$ on $W$, where $\omega=d \beta$ is a positive symplectic form on $W$ and the Liouville vector field $Y$ given by $i_{Y}(\omega)=\beta$ is positively transverse to $\partial W$. It follows that the 1 -form $\beta_{0}=\left.\beta\right|_{\partial W}$ (this notation means $\beta$ pulled back to $\partial W$ ) is a positive contact form with kernel $\zeta$.

We now recall the definition of a sutured contact manifold.

Definition 2.2. A compact oriented $2 n+1$-dimensional manifold $M$ with boundary and corners is a sutured contact manifold if it comes with an oriented, not necessarily 
connected submanifold $\Gamma \subset \partial M$ of dimension $2 n-1$ (called the suture), together with a neighborhood $U(\Gamma)=[-1,0] \times[-1,1] \times \Gamma$ of $\Gamma=\{0\} \times\{0\} \times \Gamma$ in $M$, with coordinates $(\tau, t) \in[-1,0] \times[-1,1]$, such that the following holds:

(1) $U \cap \partial M=(\{0\} \times[-1,1] \times \Gamma) \cup([-1,0] \times\{-1\} \times \Gamma) \cup([-1,0] \times\{1\} \times \Gamma)$.

(2) $\partial M \backslash(\{0\} \times(-1,1) \times \Gamma)=R_{-}(\Gamma) \sqcup R_{+}(\Gamma)$, where the orientation of $\partial M$ agrees with that of $R_{+}(\Gamma)$ and is opposite that of $R_{-}(\Gamma)$ and the orientation of $\Gamma$ agrees with the boundary orientation of $R_{+}(\Gamma)$.

(3) The corners of $M$ are precisely $\{0\} \times\{ \pm 1\} \times \Gamma$.

In addition, $M$ is equipped with a contact structure $\xi$, which is given by the kernel of a positive contact 1-form $\alpha$ such that

(i) $\left(R_{ \pm}(\Gamma), \beta_{ \pm}=\left.\alpha\right|_{R_{ \pm}(\Gamma)}\right)$ is a Liouville manifold;

(ii) $\alpha=C d t+\beta$ inside $U(\Gamma)$, where $C>0$ and $\beta$ is independent of $t$ and does not have a $d t$-term;

(iii) $\partial_{\tau}=Y_{ \pm}$, where $Y_{ \pm}$is a Liouville vector field for $\beta_{ \pm}$.

Such a contact form $\alpha$ is said to be adapted to $(M, \Gamma, U(\Gamma))$.

Here we briefly describe the way to glue sutured contact manifolds. This procedure was first described by Colin and Honda [2005] and then generalized by Colin et al. [2011].

Let $\left(M^{\prime}, \Gamma^{\prime}, U\left(\Gamma^{\prime}\right), \xi^{\prime}\right)$ be a sutured contact 3-manifold with an adapted contact form $\alpha^{\prime}$. We denote by $\pi$ the projection along $\partial_{t}$ defined on $U\left(\Gamma^{\prime}\right)$.

Take 2-dimensional submanifolds $P_{ \pm} \subset R_{ \pm}\left(\Gamma^{\prime}\right)$ such that $\partial P_{ \pm}$is the union of $\left(\partial P_{ \pm}\right)_{\partial} \subset \partial R_{ \pm}\left(\Gamma^{\prime}\right),\left(\partial P_{ \pm}\right)_{\text {int }} \subset \operatorname{int}\left(R_{ \pm}\left(\Gamma^{\prime}\right)\right)$ and $\partial P_{ \pm}$is positively transversal to the Liouville vector field $Y_{ \pm}^{\prime}$ on $R_{ \pm}\left(\Gamma^{\prime}\right)$. Whenever we refer to $\left(\partial P_{ \pm}\right)_{\text {int }}$ and $\left(\partial P_{ \pm}\right)_{\partial}$, we assume that closures are taken as appropriate. Moreover we make the assumption that $\left.\pi\left(\left(\partial P_{-}\right)_{\partial}\right) \cap \pi\left(\partial P_{+}\right)_{\partial}\right)=\varnothing$.

Let $\varphi$ be a diffeomorphism which sends $\left(P_{+},\left.\beta_{+}^{\prime}\right|_{P+}\right)$ to $\left(P_{-},\left.\beta_{-}^{\prime}\right|_{P_{-}}\right)$and takes $\left(\partial P_{+}\right)_{\text {int }}$ to $\left(\partial P_{-}\right)_{\partial}$ and $\left(\partial P_{+}\right)_{\partial}$ to $\left(\partial P_{-}\right)_{\text {int }}$. Note that, since $\operatorname{dim} M=3$, we only need $\left.\beta_{+}^{\prime}\right|_{P_{+}}$and $\varphi^{*}\left(\left.\beta_{-}^{\prime}\right|_{P_{-}}\right)$to match up on $\partial P_{+}$, since we can linearly interpolate between primitives of positive area forms on a surface.

Topologically, we construct the sutured manifold $(M, \Gamma)$ from $\left(M^{\prime}, \Gamma^{\prime}\right)$ and the gluing data $\left(P_{+}, P_{-}, \varphi\right)$ as follows: Let $M=M^{\prime} / \sim$, where

- $x \sim \varphi(x)$ for all $x \in P_{+}$;

- $x \sim x^{\prime}$ if $x, x^{\prime} \in \pi^{-1}\left(\Gamma^{\prime}\right)$ and $\pi(x)=\pi\left(x^{\prime}\right) \in \Gamma^{\prime}$.

Then

$$
R_{ \pm}(\Gamma)=\frac{\overline{R_{ \pm}\left(\Gamma^{\prime}\right) \backslash P_{ \pm}}}{\left(\partial P_{ \pm}\right)_{\text {int }}} \sim \pi_{ \pm}\left(\left(\partial P_{\mp}\right)_{\partial}\right)
$$


and

$$
\Gamma=\frac{\overline{\Gamma^{\prime} \backslash \pi\left(\partial P_{+} \sqcup \partial P_{-}\right)}}{\pi\left(\left(\partial P_{+}\right)_{\text {int }} \cap\left(\partial P_{+}\right)_{\partial}\right)} \sim \pi\left(\left(\partial P_{-}\right)_{\text {int }} \cap\left(\partial P_{-}\right)_{\partial}\right) .
$$

For a detailed description of the gluing procedure, see [Colin et al. 2011].

Finally, we describe the way to complete sutured contact manifold $(M, \alpha)$ to a noncompact contact manifold $\left(M^{*}, \alpha^{*}\right)$. This construction was first described in [Colin et al. 2011].

Let $(M, \Gamma, U(\Gamma), \xi)$ be a sutured contact manifold with an adapted contact form $\alpha$. The form $\alpha$ is then given by

$$
C d t+\beta_{ \pm}
$$

on collar neighborhoods $[1-\varepsilon, 1] \times R_{+}(\Gamma)$ and $[-1,-1+\varepsilon] \times R_{-}(\Gamma)$ of $R_{+}(\Gamma)=$ $\{1\} \times R_{+}(\Gamma)$ and $R_{-}(\Gamma)=\{-1\} \times R_{-}(\Gamma)$, where $t \in[-1,-1+\varepsilon] \cup[1-\varepsilon, 1]$ extends the $t$-coordinate on $U$. On $U$ we have $\alpha=C d t+\beta, \beta=\beta_{+}=\beta_{-}$and $\partial_{\tau}$ is a Liouville vector field $Y$ for $\beta$. We first extend $\alpha$ to $[1, \infty) \times R_{+}(\Gamma)$ and $(-\infty,-1] \times R_{-}(\Gamma)$ by taking $C d t+\beta_{ \pm}$as appropriate. The boundary of this new manifold is $\{0\} \times \mathbb{R} \times \Gamma$. Notice that since $\partial_{\tau}=Y$, the form $\left.d \beta\right|_{[-1,0] \times\{t\} \times \Gamma}$ is the symplectization of $\left.\beta\right|_{\{0\} \times\{t\} \times \Gamma}$ in the positive $\tau$-direction. We glue $[0, \infty) \times \mathbb{R} \times \Gamma$ with the form $C d t+e^{\tau} \beta_{0}$, where $\beta_{0}$ is the pullback of $\beta$ to $\{0\} \times\{t\} \times \Gamma$.

We denote by $M^{*}$ the noncompact extension of $M$ described above and by $\alpha^{*}$ the extension of $\alpha$ to $M^{*}$.

2B. Review of cylindrical contact homology. In this section, we review the definition of cylindrical contact homology for sutured manifolds. We refer to [Colin et al. 2011] for more details of this construction.

Let $(M, \Gamma, U(\Gamma), \xi)$ be a sutured contact manifold with an adapted contact form $\alpha$ and $\left(M^{*}, \alpha^{*}\right)$ be its completion.

The Reeb vector field $R_{\alpha^{*}}$ that is associated to a contact form $\alpha^{*}$ is given by $d \alpha^{*}\left(R_{\alpha^{*}}, \cdot\right)=0$ and $\alpha^{*}\left(R_{\alpha^{*}}\right)=1$. We assume that $R_{\alpha^{*}}$ is nondegenerate, i.e., the first return map along each (not necessarily simple) periodic orbit does not have 1 as an eigenvalue. Observe that nondegeneracy can always be achieved by a small perturbation.

Remark 2.3. Every periodic orbit of $R_{\alpha^{*}}$ lies in $M$. Hence, the set of periodic Reeb orbits of $R_{\alpha^{*}}$ coincides with the set of periodic Reeb orbits of $R_{\alpha}$.

A Reeb orbit $\gamma$ is called elliptic or positive (respectively negative) hyperbolic if the eigenvalues of $P_{\gamma}$ are on the unit circle or the positive (resp. negative) real line respectively.

If $\tau$ is a trivialization of $\xi$ over $\gamma$, we can then define the Conley-Zehnder index. In 3-dimensional situation, we can explicitly describe the Conley-Zehnder index and its behavior under multiple covers as follows: 
Proposition 2.4 [Hutchings 2002]. If $\gamma$ is elliptic, then there is an irrational number $\phi \in \mathbb{R}$ such that $P_{\gamma}$ is conjugate in $S L_{2}(\mathbb{R})$ to a rotation by angle $2 \pi \phi$ and

$$
\mu_{\tau}\left(\gamma^{k}\right)=2\lfloor k \phi\rfloor+1,
$$

where $2 \pi \phi$ is the total rotation angle with respect to $\tau$ of the linearized flow around the orbit.

If $\gamma$ is positive (respectively negative) hyperbolic, then there is an even (respectively odd) integer $r$ such that the linearized flow around the orbit rotates the eigenspaces of $P_{\gamma}$ by angle $\pi r$ with respect to $\tau$ and

$$
\mu_{\tau}\left(\gamma^{k}\right)=k r .
$$

A closed orbit of $R_{\alpha^{*}}$ is said to be good if it does not cover a simple orbit $\gamma$ an even number of times, where the first return map $\xi_{\gamma(0)} \rightarrow \xi_{\gamma(T)}$ has an odd number of eigenvalues in the interval $(-1,0)$. Here $T$ is the period of the orbit $\gamma$. An orbit that is not good is called bad.

We now recall the notion of an almost complex structure on $\mathbb{R} \times M^{*}$ that is tailored to $\left(M^{*}, \alpha^{*}\right)$.

Let $(W, \beta)$ be a Liouville manifold and $\zeta$ be the contact structure given on $\partial W$ by $\operatorname{ker}\left(\beta_{0}\right)$, where $\beta_{0}=\left.\beta\right|_{\partial W}$. In addition, An almost complex structure $J_{0}$ on $\widehat{W}$ is $\hat{\beta}$-adapted if $J_{0}$ is $\beta_{0}$-adapted on $[0, \infty) \times \partial W$; and $d \beta\left(v, J_{0} v\right)>0$ for all nonzero tangent vectors $v$ on $W$.

Definition 2.5. Let $(M, \Gamma, U(\Gamma), \xi)$ be a sutured contact manifold, $\alpha$ be an adapted contact form and $\left(M^{*}, \alpha^{*}\right)$ be its completion. We say that an almost complex structure $J$ on $\mathbb{R} \times M^{*}$ is tailored to $\left(M^{*}, \alpha^{*}\right)$ if the following conditions hold:

(1) $J$ is $\alpha^{*}$-adapted, i.e., $J$ is $\mathbb{R}$-invariant, $J(\xi)=\xi, d \alpha(v, J v)>0$ for nonzero $v \in \xi$ and $J\left(\partial_{s}\right)=R_{\alpha^{*}}$, where $s$ denotes the $\mathbb{R}$-coordinate.

(2) $J$ is $\partial_{t}$-invariant in a neighborhood of $M^{*} \backslash \operatorname{int}(M)$.

(3) The projection of $J$ to $T \widehat{R_{ \pm}(\Gamma)}$ is a $\hat{\beta}_{ \pm}$-adapted almost complex structure $J_{0}$ on the completion $\left(\widehat{R_{+}(\Gamma)}, \hat{\beta}_{+}\right) \sqcup\left(\widehat{R_{-}(\Gamma)}, \hat{\beta}_{-}\right)$of the Liouville manifold $\left(R_{+}(\Gamma), \beta_{+}\right) \sqcup\left(R_{-}(\Gamma), \beta_{-}\right)$. Moreover, the flow of $\partial_{t}$ identifies $\left.J_{0}\right|_{\widehat{R_{+}}(\Gamma) \backslash R_{+}(\Gamma)}$ and $J_{0} \mid \widehat{R_{-}(\Gamma) \backslash R_{-}(\Gamma)}$.

Given a sutured contact manifold $(M, \Gamma, U(\Gamma), \alpha)$ and an $\alpha^{*}$-adapted almost complex structure $J$, we define the sutured cylindrical contact homology group $H C^{\text {cyl }}(M, \Gamma, \alpha, J)$ to be the cylindrical contact homology of $\left(M^{*}, \alpha^{*}, J\right)$. The cylindrical contact homology chain complex $C(\alpha, J)$ is a $\mathbb{Q}$-module freely generated by all good Reeb orbits, where the grading $|\cdot|$ and the boundary map $\partial$ are defined as in [Bourgeois 2009] with respect to the $\alpha^{*}$-adapted almost complex structure 
$J$. The homology of $C(\alpha, J)$ is the sutured cylindrical contact homology group $H C^{\mathrm{cyl}}(M, \Gamma, \alpha, J)$.

For our calculations we need the following construction of a "global" symplectic trivialization described in [Bourgeois 2009]. Assume that all the Reeb orbits of $R_{\alpha}$ are good. Let us now choose trivializations $\tau(\gamma)$ consistently for all Reeb orbits $\gamma$. Assume that $H_{1}(M ; \mathbb{Z})$ is a free module. We pick representatives $C_{1}, \ldots, C_{s}$ in $H_{1}(M ; \mathbb{Z})$ for a basis of $H_{1}(M ; \mathbb{Z})$, together with a trivialization of $\xi$ along each representative $C_{i}, i=1, \ldots, s$. Now for a Reeb orbit $\gamma$, we distinguish the following cases:

(1) $[\gamma]=0 \in H_{1}(M ; \mathbb{Z})$. Choose a spanning surface $S_{\gamma}$ and use it to trivialize $\xi$ along $\gamma$.

(2) $0 \neq[\gamma] \in H_{1}(M ; \mathbb{Z})$. We choose a surface $S_{\gamma}$ realizing a homology between $\gamma$ and a linear combination of the representatives $C_{i}, i=1, \ldots, s$. We then use $S_{\gamma}$ to extend the chosen trivializations of $\xi$ along the $C_{i}$ to $\gamma$.

We denote the obtained trivialization by $\tau$.

To a $J$-holomorphic cylinder in $\mathcal{M}^{J}\left(\gamma ; \gamma^{\prime}\right)$, we can glue the chosen surfaces $S_{\gamma}$ and $S_{\gamma^{\prime}}$ and obtain a closed surface in $M$ (here $M^{J}\left(\gamma ; \gamma^{\prime}\right)$ is a moduli space of $J$-holomorphic cylinders considered in cylindrical contact homology theory). Let $A \in H_{2}(M ; \mathbb{Z})$ be its homology class; we can use it to decorate the corresponding connected component $M_{A}^{J}\left(\gamma ; \gamma^{\prime}\right)$ of the moduli space. Using $\tau$ we can write

$$
\operatorname{ind}(u)=|\gamma|-\left|\gamma^{\prime}\right|+2\left\langle c_{1}(\xi), A\right\rangle
$$

for $u \in \mathcal{M}_{A}^{J}\left(\gamma ; \gamma^{\prime}\right)$, where $|\gamma|$ is the Conley-Zehnder grading of $\gamma$ defined by

$$
|\gamma|:=\mu_{\tau}(\gamma)-1 \text {. }
$$

We will use (2B.1) and (2B.2) for our calculations.

In addition, we will need the following fact, which is a consequence of Lemma 5.4 in [Bourgeois et al. 2003]:

Fact 2.6. Let $(M, \alpha)$ be a closed, oriented contact manifold with nondegenerate Reeb orbits and $u \in \mathcal{M}^{J}\left(\gamma ; \gamma^{\prime}\right)$, where $\gamma$ and $\gamma^{\prime}$ are good Reeb orbits and $J$ is an $\alpha$-adapted almost complex structure on $\mathbb{R} \times M$. Then $\mathscr{A}(\gamma):=\int_{\gamma} \alpha \geq \int_{\gamma^{\prime}} \alpha=: \mathscr{A}\left(\gamma^{\prime}\right)$ with equality if and only if $\gamma=\gamma^{\prime}$ and in this case the moduli space consists of a single element $\mathbb{R} \times \gamma$.

Theorem 2.7 [Bourgeois 2009]. Let $(M, \alpha)$ be a closed, oriented contact manifold with nondegenerate Reeb orbits. Let $C_{m}^{h}(M, \alpha)$ be the cylindrical contact homology complex, where $h$ is a homotopy class of Reeb orbits and $m$ corresponds to the Conley-Zehnder grading. If $C_{k}^{0}(M, \alpha)=0$ for $k=-1,0,1$, we have for every free homotopy class $h$ : 
(1) $\partial^{2}=0$.

(2) $H\left(C_{*}^{h}(M, \alpha), \partial\right)$ is independent of the contact form $\alpha$ for $\xi$, the almost complex structure $J$ and the choice of perturbation for the moduli spaces.

When $M$ is closed and $\mathbb{R} \times M$ is 4-dimensional, the following transversality result has been proven by Momin [2011, Proposition 2.10]:

Theorem 2.8 [Momin 2011]. Let $u \in M^{J}\left(\gamma ; \gamma^{\prime}\right)$ be such that $\operatorname{ind}(u)=1$. Then the linearization of the Cauchy-Riemann operator is surjective at $u$.

Remark 2.9. Theorem 2.8 does not require $J$ to be generic. In addition, note that Theorem 2.8 can be considered as a consequence of the automatic transversality result of Wendl [2010, Theorem 0.1].

Finally, we recall the following result of Colin, Ghiggini, Honda and Hutchings:

Theorem 2.10 [Colin et al. 2011]. Let $(M, \Gamma, U(\Gamma), \xi)$ be a sutured contact 3manifold with an adapted contact form $\alpha,\left(M^{*}, \alpha^{*}\right)$ be its completion and $J$ be an almost complex structure on $\mathbb{R} \times M^{*}$ which is tailored to $\left(M^{*}, \alpha^{*}\right)$. Then the contact homology algebra $\mathrm{HC}(M, \Gamma, \xi)$ is defined and independent of the choice of contact 1 -form $\alpha$ with $\operatorname{ker}(\alpha)=\xi$, adapted almost complex structure $J$, and abstract perturbation.

Remark 2.11. Fact 2.6, Theorems 2.7 and 2.8 and formulas (2B.1) and (2B.2) hold for $J$-holomorphic curves in the symplectization of the completion of a sutured contact manifold, provided that we choose the almost complex structure $J$ on $\mathbb{R} \times M^{*}$ to be tailored to $\left(M^{*}, \alpha^{*}\right)$.

Remark 2.12. Theorem 2.10 and Remark 2.11 rely on the assumption that the machinery, needed to prove the analogous properties for contact homology and cylindrical contact homology in the closed case, works.

\section{Construction}

The goal of this section is to construct the sutured contact solid torus $\left(S^{1} \times D^{2}, \tilde{\Gamma}, \tilde{\alpha}_{\delta}\right)$, where $\tilde{\Gamma}$ consists of $2 n$ parallel sutures of slope $-k / l,(k, l)=1, k>l>0$ and $n \in \mathbb{N}$. Here $\tilde{\alpha}_{\delta}$ is a contact form such that $\xi=\operatorname{ker} \tilde{\alpha}_{\delta}$ is a universally tight contact structure and the set of embedded orbits of $R_{\tilde{\alpha}_{\delta}}$ consists of an elliptic orbit $\gamma$ and hyperbolic orbits $\gamma_{1}, \ldots, \gamma_{n}$ with

$$
\begin{aligned}
{[\gamma] } & =1, & \mu_{\tau}\left(\gamma_{i}^{s}\right) & =-2 l s, \quad \mathscr{A}\left(\gamma^{k}\right)>\mathscr{A}\left(\gamma_{i}\right), \\
{\left[\gamma_{i}\right] } & =k \in \mathbb{Z} \simeq H_{1}\left(S^{1} \times D^{2} ; \mathbb{Z}\right), & \mu_{\tau}\left(\gamma^{t}\right) & =-2 m l+1,
\end{aligned}
$$

where $(m-1) k<t \leq m k$, for some "global" symplectic trivialization $\tau$. Here $i=1, \ldots, n, t \leq N_{\delta}, s \leq N_{\delta} / k, N_{\delta} \gg 0$. 
3A. Gluing map. First we construct $H \in C^{\infty}\left(\mathbb{R}^{2}\right)$. The time-1 flow of the Hamiltonian vector field associated to $H$ composed with an appropriate rotation will play a role of the gluing map when we will apply the gluing construction described in Section 2A to the sutured contact solid cylinder constructed in Section 3B.

We fix $p \in \mathbb{R}^{2}$ and consider $H_{\text {sing }}: \mathbb{R}^{2} \rightarrow \mathbb{R}$ given by $H_{\text {sing }}=\mu r^{2} \cos (n k \theta)$ in polar coordinates $(r, \theta)$ about $p$, where $\mu>0, n \geq 1$ and $k \in \mathbb{N} \backslash\{1\}$. Note that $H_{\text {sing }}$ is singular only at $p$.

Lemma 3.1. There exists a function $H \in C^{\infty}\left(\mathbb{R}^{2}\right)$ which satisfies the following properties:

- $H=H_{\text {sing }}$ on $\mathbb{R}^{2} \backslash D\left(r_{\text {sing }}\right)$ for some $r_{\text {sing }}>0$.

- $H$ is $\frac{2 \pi}{n k}$-symmetric with respect to $\theta$.

- The set of critical points of $H$ consists of equally spaced saddle points $p_{1}, \ldots, p_{n k}$ and a critical point $p$.

- There exists a neighborhood $U_{s}$ of $p_{s}$ with coordinates $(x, y)$ such that $H=$ axy on $U_{s}$ with $a>0$, and such that $\frac{2 \pi}{n k}$-rotation about $p$ that we call $R_{n k}$ maps $U_{s}$ with the corresponding coordinate system to $U_{s+1}$ with the corresponding coordinate system for $s=1, \ldots, n k$.

- There exists a neighborhood $U$ of $p$ such that $H=\tilde{B} r^{2}-\tilde{C}$ on $U$, where $\tilde{C}>0$ and $\tilde{B}$ is a small positive number.

Proof. We construct $H \in C^{\infty}\left(\mathbb{R}^{2}\right)$ from $H_{\text {sing }}$ by perturbing $H_{\text {sing }}$ on a disk $D\left(r_{\text {sing }}\right)$ about $p$ in such a way that $H$ has $n k$ equally spaced saddle points, critical point at $p$ and interpolates with no other critical points with $H_{\text {sing. }}$. In other words, $H=H_{\text {sing }}$ on $\mathbb{R}^{2} \backslash D\left(r_{\text {sing }}\right)$ for some $r_{\text {sing }}>0$. For the level sets of $H_{\text {sing }}$ and $H$ in the case $n=1, k=3$ we refer to Figure 1 .

The construction of $H$ is a modification of the construction described in [CottonClay 2009].

We proceed in four steps.

(1) We consider

$$
\begin{aligned}
H_{1} & =H_{\text {sing }}+f(r, \theta)=H_{\text {sing }}+f_{\exp }(r, \theta)+g(r, \theta) \\
& =\mu r^{2} \cos (n k \theta)-A e^{-m r^{2}}+g(r, \theta),
\end{aligned}
$$

where $A$ and $m$ are positive constants, and $g(r, \theta)$ is a smooth function to be chosen later. We are interested in the critical points of $H_{1}$ away from the origin.

We calculate

$$
\frac{\partial H_{1}}{\partial r}=2 \mu r \cos (n k \theta)+2 m r A e^{-m r^{2}}+\frac{\partial g}{\partial r}, \quad \frac{\partial H_{1}}{\partial \theta}=-n k \mu r^{2} \sin (n k \theta) .
$$



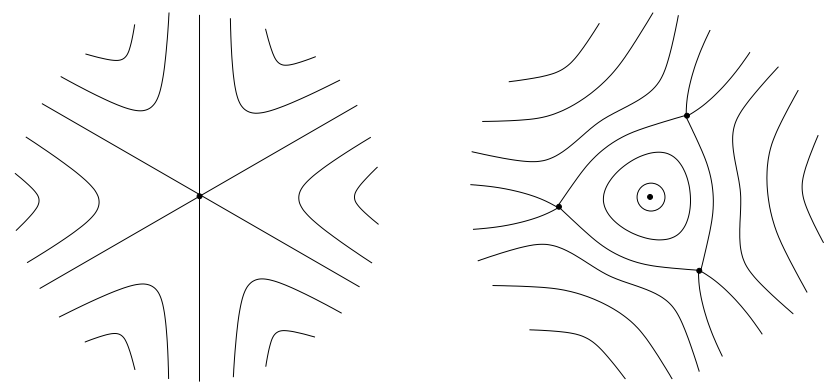

Figure 1. The level sets of $H_{\text {sing }}$ (left) and the level sets of $H$ (right) in the case $n=1, k=3$.

Thus, at the critical points of $H_{1}$ we must have $\sin (n k \theta)=0$. In this case, $\cos (n k \theta)= \pm 1$. If $\cos (n k \theta)=1$, then $\partial H_{1} / \partial r-\partial g / \partial r$ cannot be zero. When $\cos (n k \theta)=-1, \partial H_{1} / \partial r-\partial g / \partial r=-2 \mu r+2 m r A e^{-m r^{2}}$. For $r>0, \partial H_{1} / \partial r-$ $\partial g / \partial r=0$ when $e^{m r^{2}}=m A / \mu$, i.e., when

$$
r=r_{c}:=\sqrt{\frac{1}{m} \ln \frac{m A}{\mu}} .
$$

We impose the restriction that $m A>\mu$. By making $m$ large, we can make $r_{c}$ arbitrarily small. When $\cos (n k \theta)=-1, H_{1}-g(r, \theta)=-\frac{\mu}{m}(\ln (m A / \mu)+1)$. Let $g(r)$ be equal to $\frac{\mu}{m}(\ln (m A / \mu)+1)$ on the annular neighborhood of $r=r_{c}$. For such $g, H_{1}$ is 0 at the critical points, i.e., at the points $\left(r_{c}, \theta\right)$, where $\cos (n k \theta)=-1$.

In summary, we get critical points at one value of $r$ at the values of $\theta$ when $\cos (n k \theta)=-1$, that is, for $n k$ values of $\theta$. These are our $n k$ saddle points (it's not hard to see they are saddle points; alternatively, we can deduce that they must be for index reasons).

(2) Keeping $f_{\exp }$ solely a function of $r$ and keeping $g$ constant, we cut off $f_{\exp }$ smoothly starting at some point past $r_{c}$ to give a Hamiltonian $\mathrm{H}_{2}$ which agrees with $H_{\text {sing }}+g$ outside a ball. As long as $\partial f_{\exp } / \partial r<2 \mu r$, there are no new critical points.

Note that $f_{\exp }\left(r_{c}\right)=-\mu / m$. Keeping $\partial f_{\exp } / \partial r$ near $\mu r_{c}$ (which, using, e.g., $A=e \mu / m$, is $1 / \sqrt{m}$ ), we can bring $f_{\exp }$ to zero in a radial distance of a constant times $1 / \sqrt{m}$; that is, for $m$ large we can make $H_{2}$ agree with $H_{\text {sing }}+g$ outside an arbitrarily small ball.

For $A=e \mu / m, g=2 \mu / m$. Then keeping $g$ solely a function of $r$, we cut off $g(r, \theta)$ smoothly starting at some point past the point where $H_{2}=H_{\text {sing }}+g$ to give Hamiltonian $H_{3}$. As long as $\partial g / \partial r>-2 \mu r$, there are no new critical points. We can make it in such a way that $H_{3}$ agrees with $H_{\text {sing }}$ outside a small ball. 
(3) Recall that $H_{3}=H_{\text {sing }}+f_{\text {exp }}+g$ near the origin and $g(r, \theta)=2 \frac{\mu}{m}>0$. Note that $g(r, \theta)$ is small for large $m$. Now keeping $g$ constant we modify $H_{\text {sing }}+f_{\exp }+g$ near the origin to give us $H_{4}$ which is $B r^{2}-C$ near the origin (for $B>0$ ), which corresponds to the Hamiltonian flow rotating at a constant angular rate. Since

$$
\frac{\partial H_{3}}{\partial r}=\frac{\partial\left(H_{\text {sing }}+f_{\text {exp }}\right)}{\partial r}>0 \text { for } r<r_{c},
$$

we can patch together $\mathrm{Br}^{2}-\mathrm{C}$ near the origin with $\mathrm{H}_{2}$ outside a small ball of radius less than $r_{c}$ in a radially symmetric manner to get $H_{4}$ such that $\partial H_{4} / \partial r>0$ for $r<r_{c}$ (we do this by choosing $C$ sufficiently large). Note that $H_{4}$ has a critical point at the origin.

(4) Finally, to ensure no fixed points of the time-1 flow of the Hamiltonian vector field of $H$, we let $H$ be $H_{4}$ multiplied by a radially symmetric function which is $\epsilon$ for $r<R$ (for $\epsilon$ sufficiently small that the only fixed points of the time-1 flow inside radius $R$ are the critical points and for $R$ large enough that $H_{4}$ agrees with $H_{\text {sing }}$ for $r>R$ ) and 1 for $r>2 R$. This creates no new fixed points in the region $R<r<2 R$ because $H_{4}$ and $\partial H_{4} / \partial r$ have the same sign there. Now there are no fixed points of the time- 1 flow of the Hamiltonian vector field of $H$, except for the $n k+1$ critical points of $H$ because outside radius $R$ there are no compact flow lines.

Let $p_{1}, \ldots, p_{n k}$ denote the equally spaced saddle points of $H$ ordered counterclockwise, i.e., $R_{n k}\left(p_{i}\right)=p_{i+1}$, where $R_{n k}$ corresponds to the $\frac{2 \pi}{n k}$-rotation around $p$. We note that $H\left(p_{s}\right)=0$ for $s=1, \ldots, n k$. Hence, by Morse lemma (arguing the same way as in Lemma 3.2 in [Golovko 2011]) we get that there is a neighborhood $U_{s}$ of $p_{s}$ such that $H=a x y$ on $U_{s}$, where $s=1, \ldots, n k$ and $a>0$. In addition, observe that $H$ is $\frac{2 \pi}{n k}$-symmetric with respect to $\theta$. Therefore, the $U_{s}$ together with coordinates $(x, y)$ are $\frac{2 \pi}{n k}$-symmetric with respect to $\theta$, i.e., $R_{n k}\left(U_{s}\right)=U_{s+1}$ and coordinates on $U_{s}$ maps to the coordinate on $U_{s+1}$. Finally, note that $H=\tilde{B} r^{2}-\tilde{C}$ on a neighborhood of the center of $D\left(r_{\text {sing }}\right)$, which we call $U$, where $\tilde{C}>0$ and $\tilde{B}$ is a small positive number and hence Hamiltonian flow rotates at a constant rate near the origin.

3B. Sutured contact solid cylinder. In this section, we construct the sutured contact solid cylinder that we later will glue to get the sutured contact solid torus with $2 n$ sutures of slope $-k / l$, where $n \in \mathbb{N},(k, l)=1$ and $k>l>0$.

Let $\gamma_{p, p_{s}}$ be an embedded curve in $\mathbb{R}^{2}$ which starts at $p$ and ends at $p_{s}$ for $s=1, \ldots, n k$. For the time being, we can think about $\gamma_{p, p_{s}}$ as about the segment connecting $p$ and $p_{s}$. 
Lemma 3.2. There exists a 1-form $\beta$ on $\mathbb{R}^{2}$ satisfying the following:

(1) $d \beta>0$.

(2) Its singular foliation given by $\operatorname{ker} \beta$ has isolated singularities and no closed orbits.

(3) $\beta=\frac{1}{2} \varepsilon_{c} r^{2} d \theta$ on $U$ with respect to the polar coordinates whose origin is at the center of $D\left(r_{\text {sing }}\right) ; \beta=\frac{1}{2} \varepsilon_{\text {sym }}(x d y-y d x)$ on $U_{s}$ with respect to the coordinates from Lemma 3.1, where $s \in\{1, \ldots, n k\} ; \beta=\frac{1}{2} r^{2} d \theta$ on $\mathbb{R}^{2} \backslash D\left(r_{\text {sing }}\right)$ with respect to the polar coordinates whose origin is at the center of $D\left(r_{\text {sing }}\right)$; here $0<\varepsilon_{c} \ll \varepsilon_{\text {sym }} \ll 1$.

(4) The set of hyperbolic points of the singular foliation of $\beta$ is given by $\left\{q_{s}\right\}_{s=1}^{n k}$ such that $q_{s}$ lies on $\gamma_{p, p_{s}}$ outside of $U_{s}$ and $U$.

(5) $\beta$ is $\frac{2 \pi}{n k}$-symmetric, i.e., $R_{n k}^{*}(\beta)=\beta$.

Proof. Consider a singular foliation $\mathscr{F}$ on $\mathbb{R}^{2}$ which satisfies the following:

(1) $\mathscr{F}$ is Morse-Smale and has no closed orbits.

(2) The singular set of $\mathscr{F}$ consists of elliptic points and hyperbolic points. The elliptic points are the equally spaced saddle points of $H$ and the center of $D\left(r_{\text {sing }}\right)$. The set of hyperbolic points of the singular foliation of $\beta$ is given by $\left\{q_{s}\right\}_{s=1}^{n k}$ such that $q_{s}$ lies on $\gamma_{p, p_{s}}$ outside of $U_{s}$ and $U$.

(3) $\mathscr{F}$ is oriented and for one choice of orientation the flow is transverse to and exits from $\partial D\left(r_{\text {sing }}\right)$.

(4) $\mathscr{F}$ is $\frac{2 \pi}{n k}$-symmetric with respect to $\theta$.

Next, we modify $\mathscr{F}$ near each of the singular points so that $\mathscr{F}$ is given by $\beta_{0}=\frac{1}{2}(x d y-y d x)$ on $U_{s}$ with respect to the coordinates from Lemma 3.1 and $\beta_{0}=2 x d y+y d x$ near a hyperbolic point. On $\mathbb{R}^{2} \backslash D\left(r_{\text {sing }}\right), \beta_{0}=\frac{1}{2} r^{2} d \theta$ with respect to the polar coordinates whose origin is at the center of $D\left(r_{\text {sing }}\right)$. In addition, on $U, \beta_{0}=\frac{1}{2} r^{2} d \theta$ with respect to the polar coordinates whose origin is at the center of $D\left(r_{\text {sing }}\right)$. From Lemma 3.1 it follows that we can do it in such a way that the modification of $\mathscr{F}$ is still $\frac{2 \pi}{n k}$-symmetric. Finally, we get $\mathscr{F}$ given by $\beta_{0}$, which satisfies $d \beta_{0}>0$ near the singular points and on $\mathbb{R}^{2} \backslash D\left(r_{\text {sing }}\right)$. Now let $\beta=g \beta_{0}$, where $g$ is a positive function with $d g(X) \gg 0$ outside of

$$
U \cup\left(\bigcup_{s=1}^{n k} U_{s}\right) \cup\left(\mathbb{R}^{2} \backslash D\left(r_{\text {sing }}\right)\right),
$$

$\left.g\right|_{\bigcup_{s=1}^{n k} U_{s}}=\varepsilon_{\mathrm{sym}},\left.g\right|_{U}=\varepsilon_{c},\left.g\right|_{\mathbb{R}^{2} \backslash D\left(r_{\text {sing }}\right)}=1$ and $X$ is an oriented vector field for $\mathscr{F}$ (nonzero away from the singular points). Here $0<\varepsilon_{c} \ll \varepsilon_{\text {sym }} \ll 1$. Since $d \beta=d g \wedge \beta_{0}+g \wedge d \beta_{0}, d g(X) \gg 0$ guarantees that $d \beta>0$. 

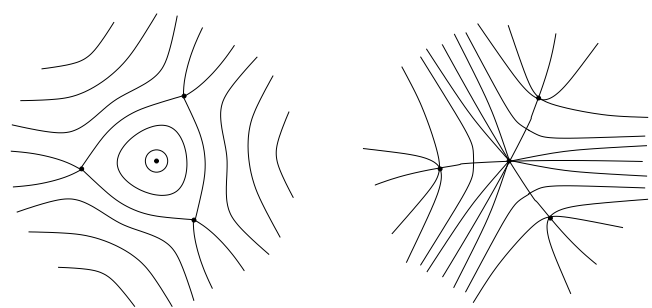

Figure 2. The level sets of $H$ (left) and the characteristic foliation of $\beta$ (right) in the case $n=1, k=3$.

For the comparison of the level sets of $H$ with the singular foliation of $\beta$ in the case $n=1, k=3$ we refer to Figure 2 .

Lemma 3.3. Let $\beta$ be a 1-form from Lemma 3.2. The Hamiltonian vector field $X_{H}$ of $H$ with respect to the area form $d \beta$ satisfies $\beta\left(X_{H}\right)=H$ on

$$
\left(\bigcup_{s=1}^{n k} U_{s}\right) \cup\left(\mathbb{R}^{2} \backslash D\left(r_{\text {sing }}\right)\right) .
$$

In addition, the Hamiltonian vector field $X_{H}$ of $H$ with respect to the area form $d \beta$ satisfies $\beta\left(X_{H}\right)-H=\tilde{C}$ on $U$.

Proof. First, Lemmas 3.1 and 3.2 imply that $\beta=\frac{1}{2} \varepsilon_{c} r^{2} d \theta, H=\tilde{B} r^{2}-\tilde{C}$ on $U$ and $\varepsilon_{c}$ is a small positive number. Now we show that $X_{H}=\frac{2 \tilde{B}}{\varepsilon_{c}} \frac{\partial}{\partial \theta}$ is a solution of $\beta\left(X_{H}\right)-H=\tilde{C}$ on $U$. We calculate

$$
\left.i_{X_{H}}(d \beta)=\left(\frac{2 \tilde{B}}{\varepsilon_{c}} \frac{\partial}{\partial \theta}\right)\right\lrcorner\left(\varepsilon_{c} r d r \wedge d \theta\right)=-2 \tilde{B} r d r=-d H
$$

and

$$
\beta\left(X_{H}\right)-H=\frac{1}{2} \varepsilon_{c} r^{2} d \theta\left(\frac{2 \tilde{B}}{\varepsilon_{c}} \frac{\partial}{\partial \theta}\right)-\tilde{B} r^{2}+\tilde{C}=\tilde{C} .
$$

Next, we work on $U_{s}$, where $s=1, \ldots, n k$. From Lemmas 3.1 and 3.2 it follows that $\beta=\frac{1}{2} \varepsilon_{\text {sym }}(x d y-y d x)$ and $H=a x y$ on $U_{s}$. Let $X_{H}$ be a Hamiltonian vector field defined by $i_{X_{H}} d \beta=-d H$.

We show that

$$
X_{H}=-\frac{a x}{\varepsilon_{\mathrm{sym}}} \frac{\partial}{\partial x}+\frac{a y}{\varepsilon_{\mathrm{sym}}} \frac{\partial}{\partial y}
$$

is a solution of the equation

$$
\beta\left(X_{H}\right)=H
$$

on $U_{s}$. We calculate

$$
\left.i_{X_{H}}(d \beta)=\left(-\frac{a x}{\varepsilon_{\mathrm{sym}}} \frac{\partial}{\partial x}+\frac{a y}{\varepsilon_{\mathrm{sym}}} \frac{\partial}{\partial y}\right)\right\lrcorner\left(\varepsilon_{\mathrm{sym}} d x \wedge d y\right)=-a x d y-a y d x=-d H
$$


and

$$
\beta\left(X_{H}\right)=\frac{1}{2} \varepsilon_{\mathrm{sym}}(x d y-y d x)\left(-\frac{a x}{\varepsilon_{\mathrm{sym}}} \frac{\partial}{\partial x}+\frac{a y}{\varepsilon_{\mathrm{sym}}} \frac{\partial}{\partial y}\right)=a x y=H .
$$

Finally, Lemmas 3.1 and 3.2 say that $\beta=\frac{1}{2} r^{2} d \theta$ and $H=\mu r^{2} \cos (n k \theta)$ on $\mathbb{R}^{2} \backslash D\left(r_{\text {sing }}\right)$. As in the previous case, we show that

$$
X_{H}=n k \mu r \sin (n k \theta) \frac{\partial}{\partial r}+2 \mu \cos (n k \theta) \frac{\partial}{\partial \theta}
$$

is a solution of Equation (3B.1) on $\mathbb{R}^{2} \backslash D\left(r_{\text {sing }}\right)$.

We calculate

$$
\begin{aligned}
i_{X_{H}}(d \beta) & \left.=\left(n k \mu r \sin (n k \theta) \partial_{r}+2 \mu \cos (n k \theta) \partial_{\theta}\right)\right\lrcorner(r d r \wedge d \theta) \\
& =-2 \mu r \cos (n k \theta) d r+n k \mu r^{2} \sin (n k \theta) d \theta=-d H,
\end{aligned}
$$

and

$$
\begin{aligned}
\beta\left(X_{H}\right) & =\frac{1}{2} r^{2} d \theta\left(n k \mu r \sin (n k \theta) \frac{\partial}{\partial r}+2 \mu \cos (n k \theta) \frac{\partial}{\partial \theta}\right) \\
& =\mu r^{2} \cos (n k \theta)=H .
\end{aligned}
$$

Let $X_{H}$ be the Hamiltonian vector field of $H$ with respect to $d \beta$ and $\varphi_{X_{H}}^{s}$ be the time-s flow of $X_{H}$. Now we introduce the following notations:

$$
\begin{aligned}
S & :=\left\{x \in \mathbb{R}^{2} \backslash D\left(r_{\text {sing }}\right) \mid \varphi_{X_{H}}^{s}(x) \in \mathbb{R}^{2} \backslash D\left(r_{\text {sing }}\right) \text { for all } s \in[0,1]\right\}, \\
V & :=\left\{x \in U \mid \varphi_{X_{H}}^{s}(x) \in U \text { for all } s \in[-1,1]\right\}, \\
V_{i} & :=\left\{x \in U_{i} \mid \varphi_{X_{H}}^{s}(x) \in U_{i} \text { for all } s \in[-1,1]\right\} .
\end{aligned}
$$

For simplicity, let us denote $\varphi_{X_{H}}:=\varphi_{X_{H}}^{1}$.

Remark 3.4. Using the form of $X_{H}$ on $U_{i}$, where $i=1, \ldots, n k$, we may assume that the curves $\gamma_{p, p_{i}}$ in Lemma 3.2 satisfy the following list of properties:

(1) $\gamma_{p, p_{i}}$ is an embedded curve which starts at $p$ and ends at $p_{i}$;

(2) $\gamma_{p, p_{i}}$ is a part of one of the curves of the singular foliation given by $\operatorname{ker} \beta$;

(3) $\gamma_{p, p_{i}}$ coincides with one of the level sets of $H$ on $V_{i}$ and near $p_{i}$ can be presented as $W^{s}\left(\varphi_{X_{H}}, p_{i}\right)=\left\{x \mid\left(\varphi_{X_{H}}\right)^{n}(x) \rightarrow p\right.$ as $\left.n \rightarrow \infty\right\}$.

Recall that the following claim was proven in [Golovko 2011]:

Claim 3.5. If $(M, \omega)$ is an exact symplectic manifold, i.e., $\omega=d \beta$, then the flow $\varphi_{X_{H}}^{t}$ of a Hamiltonian vector field $X_{H}$ consists of exact symplectic maps, i.e.,

$$
\left(\varphi_{X_{H}}^{t}\right)^{*} \beta-\beta=d f_{t},
$$


where

$$
f_{t}=\int_{0}^{t}\left(-H+\beta\left(X_{H}\right)\right) \circ \varphi_{X_{H}}^{s} d s .
$$

Remark 3.6. From Lemma 3.3 and Claim 3.5 it follows that

$$
\varphi_{X_{H}}^{*}(\beta)-\beta=d h,
$$

where $h:=f_{1}=0$ on $S \cup \bigcup_{i=1}^{n k} V_{i}$ and $h=\tilde{C}>0$ on $V$. Hence, we get $\varphi_{X_{H}}^{*}(\beta)=\beta$ on $S \cup V \cup \bigcup_{i=1}^{n k} V_{i}$.

Now we define $\varphi_{-k / l}:=R_{-k / l} \circ \varphi_{X_{H}}$, where $R_{-k / l}: \mathbb{R}^{2} \rightarrow \mathbb{R}^{2}$ is a rotation by $-2 \pi l / k$ around $p$.

Remark 3.7. Since $R_{n k}^{*}(\beta)=\beta$, we get $R_{-k / l}^{*}(\beta)=\beta$ and hence

$$
\varphi_{-k / l}^{*}(\beta)=\left(R_{-k / l} \circ \varphi_{X_{H}}\right)^{*}(\beta)=\varphi_{X_{H}}^{*}\left(R_{-k / l}^{*}(\beta)\right)=\varphi_{X_{H}}^{*}(\beta) .
$$

Fix $R_{*} \gg r_{\text {sing }}$ such that there is an annular neighborhood $V_{R_{*}}$ of $\partial D\left(R_{*}\right)$ in $\mathbb{R}^{2}$ with $V_{R_{*}} \subset S$. Consider $D\left(R_{*}\right)$ with

$$
\beta_{0}:=\left.\beta\right|_{D\left(R_{*}\right)} \quad \text { and } \quad \beta_{1}:=\left.\varphi_{X_{H}}^{*}(\beta)\right|_{D\left(R_{*}\right)}\left(=\left.\varphi_{-k / l}^{*}(\beta)\right|_{D\left(R_{*}\right)}\right) .
$$

Note that

$$
d \beta_{1}=d\left(\left.\varphi_{X_{H}}^{*}(\beta)\right|_{D\left(R_{*}\right)}\right)=\left.\varphi_{X_{H}}^{*}(d \beta)\right|_{D\left(R_{*}\right)}=\left.(d \beta)\right|_{D\left(R_{*}\right)}=d \beta_{0}>0 .
$$

In addition, from the definitions of $V\left(R_{*}\right)$ and $D\left(R_{*}\right)$ it follows that

$$
\beta_{0}=\beta_{1} \quad \text { on } \quad V_{R_{*}} \cap D\left(R_{*}\right) .
$$

Now we recall the construction of the contact 1 -form on $[-1,1] \times D^{2}$.

Lemma 3.8 [Golovko 2011, Lemma 3.10]. Let $\beta_{0}$ and $\beta_{1}$ be two 1-forms on $D^{2}$ such that $\beta_{0}=\beta_{1}$ in a neighborhood of $\partial D^{2}$ and $d \beta_{0}=d \beta_{1}=\omega>0$. Then there exists a contact 1 -form $\alpha$ and a Reeb vector field $R_{\alpha}$ on $[-1,1] \times D^{2}$ with coordinates $(t, x)$, where $t$ is a coordinate on $[-1,1]$ and $x$ is a coordinate on $D^{2}$, with the following properties:

(1) $\alpha=d t+\varepsilon \beta_{0}$ in a neighborhood of $\{-1\} \times D^{2}$.

(2) $\alpha=d t+\varepsilon \beta_{1}$ in a neighborhood of $\{1\} \times D^{2}$.

(3) $R_{\alpha}$ is collinear to $\partial / \partial t$ on $[-1,1] \times D^{2}$.

(4) $R_{\alpha}=\partial / \partial t$ in a neighborhood of $[-1,1] \times \partial D^{2}$.

Here $\varepsilon$ is a small positive number.

In addition, recall that

$$
\alpha=\left(1+\varepsilon \chi_{1}(t) h\right) d t+\varepsilon\left(\left(1-\chi_{0}(t)\right) \beta_{0}+\chi_{0}(t) \beta_{1}\right),
$$


where $h \in C^{\infty}\left(D^{2}\right)$ such that $\beta_{1}-\beta_{0}=d h ; \chi_{0}:[-1,1] \rightarrow[0,1]$ is a smooth map for which $\chi_{0}(t)=0$ for $-1 \leq t \leq-1+\varepsilon_{\chi_{0}}, \chi_{0}(t)=1$ for $1-\varepsilon_{\chi_{0}} \leq t \leq 1, \chi_{0}^{\prime}(t) \geq 0$ for $t \in[-1,1]$ and $\varepsilon_{\chi_{0}}$ is a small positive number; $\chi_{1}(t):=\chi_{0}^{\prime}(t) ; \varepsilon$ is a sufficiently small positive number.

Remark 3.9. Note that $d \alpha=\varepsilon \omega$, where $\alpha$ is a 1-form given by (3B.4) and $\omega=$ $d \beta_{0}=d \beta_{1}>0$ on $D^{2}$.

Observe that from (3B.2) and (3B.3) it follows that $\beta_{0}$ and $\beta_{1}$ described above satisfy the conditions of Lemma 3.8. We now take $[-1,1] \times D\left(R_{*}\right)$ equipped with the contact 1-form $\alpha$ given by (3B.4). For simplicity, let us denote $\beta_{-}:=\varepsilon \beta_{0}$ and $\beta_{+}:=\varepsilon \beta_{1}$, where $\varepsilon$ is a constant from Lemma 3.8 which makes $\alpha$ contact.

3C. Gluing. We now construct $P_{+}, P_{-}$and $D$ in the way described in [Golovko 2011]. Recall that

$$
P_{+}, P_{-}, D \subset D\left(R_{*}\right) \subset \mathbb{R}^{2}
$$

are surfaces with boundary which satisfy the following properties:

(1) $P_{ \pm} \subset D$.

(2) $\left(\partial P_{ \pm}\right)_{\partial} \subset \partial D$ and $\left(\partial P_{ \pm}\right)_{\text {int }} \subset \operatorname{int}(D)$.

(3) $\varphi_{X_{H}}$ maps $P_{+}$to $P_{-}$taking $\left(\partial P_{+}\right)_{\text {int }}$ onto $\left(\partial P_{-}\right)_{\partial}$ and $\left(\partial P_{+}\right)_{\partial}$ onto $\left(\partial P_{-}\right)_{\text {int }}$.

(4) $\left(\partial P_{-}\right)_{\partial} \cap\left(\partial P_{+}\right)_{\partial}=\varnothing$.

Note that

- $\partial P_{+}=\left(\bigcup_{s=0}^{n k-1} a_{s}^{+}\right) \cup\left(\bigcup_{s=0}^{n k-1} b_{s}^{+}\right)$,

- $\partial P_{-}=\left(\bigcup_{s=0}^{n k-1} a_{s}^{-}\right) \cup\left(\bigcup_{s=0}^{n k-1} b_{s}^{-}\right)$,

- $\partial D=\left(\bigcup_{s=0}^{n k-1} a_{s}^{+}\right) \cup\left(\bigcup_{s=0}^{n k-1} b_{s}^{-}\right) \cup\left(\bigcup_{s=0}^{n k-1} c_{s}^{+}\right) \cup\left(\bigcup_{s=0}^{n k-1} c_{s}^{-}\right)$.

See Figure 3 for the schematic visualization of $P_{+}$(bounded by the bold line), $P_{-}$and $D$. For more details of this construction we refer to [Golovko 2011].

Remark 3.10. Note that the $a_{i}^{ \pm}, b_{i}^{ \pm}$and $c_{i}^{ \pm}$are constructed in such a way that

$$
a_{i}^{ \pm}, b_{i}^{ \pm}, c_{i}^{ \pm} \subset D\left(R_{*}\right) \cap S
$$

for $i=0, \ldots, n k-1$. Hence, we see that $\partial P_{+}, \partial P_{-}, \partial D \subset D\left(R_{*}\right) \cap S$. In addition, $R_{n k}\left(a_{i}^{ \pm}\right)=a_{i+1}^{ \pm}$and $R_{n k}\left(b_{i}^{ \pm}\right)=b_{i+1}^{ \pm}$, where $i, i+1$ are considered modulo $n k$.

We take $[-1,1] \times D$ with a contact form $\alpha:=\left.\alpha\right|_{[-1,1] \times D}$. Let $\Gamma=\{0\} \times \partial D$ in $[-1,1] \times D$ and $U(\Gamma):=[0,1] \times[-1,1] \times \Gamma$ be a neighborhood of $\Gamma$ with coordinates $(\tau, t) \in[0,1] \times[-1,1]$, where $t$ is a usual $t$-coordinate on $[-1,1] \times D$. From the definition of $S$ and Remark 3.10 it follows that we may assume that $U(\Gamma) \subset[-1,1] \times(S \cap D)$. 


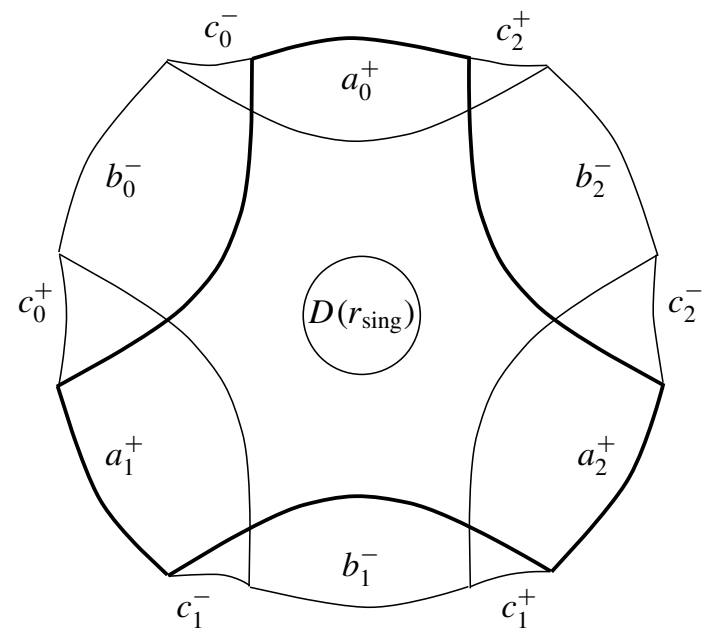

Figure 3. Construction of $P_{+}, P_{-}$and $D$ in the case $n=1, k=3$.

Lemma 3.11. $([-1,1] \times D, \Gamma, U(\Gamma), \xi)$ is a sutured contact manifold and $\alpha$ is an adapted contact form.

Proof. First note that $\left.\alpha\right|_{R_{-}}=\beta_{-}$and $\left.\alpha\right|_{R_{+}}=\beta_{+}$. Let us check that $\left(R_{-}, \beta_{-}\right)$ and $\left(R_{+}, \beta_{+}\right)$are Liouville manifolds. From the construction of $\beta_{ \pm}$it follows that $d\left(\beta_{-}\right)=d\left(\beta_{+}\right)>0$. Since $\beta_{-}=\beta_{+}$on $D \cap S$ and by (3B.4), $\alpha=d t+\beta_{-}$on $U(\Gamma)$. Recall that $\beta_{-}=\beta_{+}=\frac{1}{2} \varepsilon r^{2} d \theta$ on $D \cap S$. Hence, $\left.\alpha\right|_{U(\Gamma)}=d t+\frac{1}{2} \varepsilon r^{2} d \theta$. The calculation

$$
\left.i_{\left.Y_{ \pm}\right|_{R_{ \pm} \cap U(\Gamma)}}\left(d \beta_{ \pm}\right)=\left(\frac{1}{2} r \partial_{r}\right)\right\lrcorner(\varepsilon r d r \wedge d \theta)=\frac{1}{2} \varepsilon r^{2} d \theta=\beta_{ \pm}
$$

implies that the Liouville vector fields $\left.Y_{ \pm}\right|_{R_{ \pm} \cap U(\Gamma)}$ are equal to $\frac{1}{2} r \partial_{r}$. From the construction of $D$ it follows that $Y_{ \pm}$is positively transverse to $\partial R_{ \pm}$. Thus, $\left(R_{-}, \varepsilon \beta_{0}\right)$ and $\left(R_{+}, \varepsilon \beta_{1}\right)$ are Liouville manifolds. As already mentioned, we have $\alpha=d t+\beta_{-}$ on $U(\Gamma)$. Finally, if we take $\tau$ such that $\partial_{\tau}=\frac{1}{2} r \partial_{r}$, then $([-1,1] \times D, \Gamma, U(\Gamma), \xi)$ becomes a sutured contact manifold with an adapted contact form $\alpha$.

Then we use $\varphi_{-k / l}$ for the gluing construction. Note that $\varphi_{X_{H}}$ maps $a_{s}^{+}$to $a_{s}^{-}$ and $b_{s}^{+}$to $b_{s}^{-}$. Hence, using Remark 3.10, we see that $\varphi_{-k / l}$ maps $a_{s}^{+}$to $a_{s-n l}^{-}$and $b_{s}^{+}$to $b_{s-n l}^{-}$. Then we follow the gluing procedure briefly described in Section $2 \mathrm{~A}$ and completely written in [Colin et al. 2011]. Finally, we get a sutured contact solid torus $\left(S^{1} \times D^{2}, \tilde{\Gamma}, U(\tilde{\Gamma})\right)$ with a contact form $\tilde{\alpha}_{\delta}$, where $\tilde{\Gamma}$ is a set of $2 n$ parallel closed curves of slope $-k / l$, where $n \in \mathbb{N},(k, l)=1, k>l>0$ and $\delta$ is the rotation angle of the map $\varphi_{X_{H}}$ near $p$.

Remark 3.12. We have constructed $\left(S^{1} \times D^{2}, \tilde{\Gamma}, U(\tilde{\Gamma})\right)$ using the gluing construction for sutured manifolds. However, since there is a close connection between 
sutured contact manifolds and contact manifolds with convex boundary, we observe that the gluing construction we used for the sutured contact solid cylinder corresponds to the gluing construction for the contact 3-ball with convex boundary and one dividing curve on the boundary. The corresponding gluing construction for the contact 3-ball with convex boundary corresponds (is inverse) to the convex decomposition of the contact solid torus $S^{1} \times D^{2}$ with convex boundary with respect to the convex meridional disk $\{p t\} \times D^{2}$ with $\partial$-parallel dividing curves. Hence, the constructed sutured contact solid tori are universally tight sutured contact manifolds by the gluing/classification result from Section 2 in [Honda 2002] (more precisely, Corollary 2.3, Theorem 2.5 and Corollary 2.6).

3D. Reeb orbits. Note that $\left.\varphi_{-k / l}\right|_{P_{+}}$has $n$ orbits of period $k$ obtained from the equally spaced saddle points of $H$. Lemma 3.8 and the gluing procedure briefly described in Section 2A imply that these orbits correspond to the Reeb orbits, which we call $\gamma_{1}, \ldots, \gamma_{n}$ such that

$$
\left[\gamma_{s}\right]=\left[\gamma_{t}\right]=k \in H_{1}\left(S^{1} \times D^{2} ; \mathbb{Z}\right)
$$

for $s, t=1, \ldots, n$. In addition, $\left.\varphi_{-k / l}\right|_{P_{+}}$has a periodic point of period 1 , which is $p$. It corresponds to the Reeb orbit, which we call $\gamma$, such that $[\gamma]=1 \in H_{1}\left(S^{1} \times D^{2} ; \mathbb{Z}\right)$.

Lemma 3.13. $\int_{\gamma_{s}} \tilde{\alpha}_{\delta}=\int_{\gamma_{t}} \tilde{\alpha}_{\delta}$ and $k \int_{\gamma} \tilde{\alpha}_{\delta}>\int_{\gamma_{s}} \tilde{\alpha}_{\delta}$, where $s, t=1, \ldots, n$.

Proof. Let

$$
\begin{aligned}
M^{(0)} & =\left(([-1,1] \times D) \cup\left(R_{+}(\Gamma) \times[1 ; \infty)\right) \cup\left(R_{+}(\Gamma) \times(-\infty ;-1]\right)\right), \\
\tilde{M} & =M^{(0)} \backslash\left(\left(P_{+} \times(N, \infty) \cup\left(P_{-} \times(-\infty,-N)\right) .\right.\right.
\end{aligned}
$$

In addition, let $\alpha_{\tilde{M}}$ denote the contact form on $\tilde{M}$ and let $\xi_{\tilde{M}}$ denote the contact structure defined by $\alpha_{\tilde{M}}$.

Consider $[-1,1] \times D \subset \tilde{M}$. From the construction of $\alpha$ it follows that $\beta_{+}=\beta_{-}$on $V_{s}$ and $\left.\alpha\right|_{[-1,1] \times V_{s}}=d t+\beta_{-}$for $s=1, \ldots, n k$. Hence, since the contact structure on $[1, \infty) \times P_{+}$is given by $d t+\beta_{+}$and the contact structure on $(-\infty,-1] \times P_{-}$is given by $d t+\beta_{-},\left.\alpha_{\tilde{M}}\right|_{[-N, N] \times V_{s}}=d t+\beta_{-}$on $[-N, N] \times V_{s} \subset \tilde{M}$ for $s=1, \ldots, n k$. Therefore, we get

$$
\int_{[-N, N] \times\left\{p_{s}\right\}} \alpha_{\tilde{M}}=2 N
$$

for $s=1, \ldots, n k$. From the gluing construction and (3D.1) it follows that

$$
\int_{\gamma_{s}} \tilde{\alpha}_{\delta}=2 N k
$$

for $s=1, \ldots, n$. Note that $\int_{\gamma_{s}} \tilde{\alpha}_{\delta}$ does not depend on $s$. Hence, $\int_{\gamma_{s}} \tilde{\alpha}_{\delta}=\int_{\gamma_{t}} \tilde{\alpha}_{\delta}$ for $s, t=1, \ldots, n$. 
Now from the fact that $\alpha=\left(1+\varepsilon \chi_{1}(t) h\right) d t+\beta_{-}$on $[-1,1] \times V$, where $h>0$ and $\chi_{1}(t)>0$, we get that

$$
R_{\alpha}=\frac{1}{1+\varepsilon \chi_{1}(t) h} \frac{\partial}{\partial t}
$$

on $[-1,1] \times V$. Hence, from the gluing construction we obtain $k \int_{\gamma} \tilde{\alpha}_{\delta}>2 N k$. Thus,

$$
\int_{\gamma_{s}} \tilde{\alpha}_{\delta}=\int_{\gamma_{t}} \tilde{\alpha}_{\delta} \text { and } k \int_{\gamma} \tilde{\alpha}_{\delta}>\int_{\gamma_{s}} \tilde{\alpha}_{\delta}
$$

where $s, t=1, \ldots, n$.

Lemma 3.14. All closed orbits of $R_{\tilde{\alpha}_{\delta}}$ are nondegenerate. Moreover, $\gamma$ is an elliptic orbit and $\gamma_{i}$ is a hyperbolic orbit such that $\gamma^{t}$ and $\gamma_{i}^{s}$ are good orbits for $i=1, \ldots n ; s, t \in \mathbb{N}$. There exists a symplectic trivialization $\tau$ of $\xi$ along $\gamma$ and the $\gamma_{i}$, constructed in the consistent way as described in Section $2 B$, and $N_{\delta} \in \mathbb{N}$ such that

$$
\begin{aligned}
& \mu_{\tau}\left(\gamma_{i}^{s}\right)=-2 l s, \\
& \mu_{\tau}\left(\gamma^{t}\right)=-2 m l+1,
\end{aligned}
$$

where $(m-1) k<t \leq m k$ and $i=1, \ldots, n, t \leq N_{\delta}, s \leq N_{\delta} / k$.

Proof. For simplicity, assume that $l=1$. The general calculation can be done in the analogous way.

Fix $i=1, \ldots, n$. We first observe that $\left.H\right|_{V_{i}}=a x y$, where $a>0$ and hence

$$
\left.\varphi_{X_{H}}\right|_{V_{i}}=\left(\begin{array}{ll}
\lambda & 0 \\
0 & \lambda^{-1}
\end{array}\right)
$$

where $\lambda=e^{a} \neq 1$. Let the symplectic trivialization of $\xi_{\tilde{M}}$ along $[-N, N] \times\left\{p_{i}\right\}$ be given by the framing $\left(\lambda^{\frac{-N-t}{2 N}} \partial_{x}, \lambda^{\frac{t+N}{2 N}} \partial_{y}\right)$, where $i=1, \ldots, n k$ and $(x, y)$ are coordinates on $V_{i}$ which coincide with the coordinates on $U_{i}$ from Lemma 3.1. Since Lemma 3.1 implies that $R_{n k}$ maps coordinates on $V_{i}$ to the coordinate on $V_{i+1}$, where $i, i+1$ are considered modulo $n k$, we conclude that the symplectic trivializations of $\xi_{\tilde{M}}$ along each $[-N, N] \times\left\{p_{i+n m}\right\}$ for $m=0, \ldots, k-1$ and fixed $i=1, \ldots, n$ give rise to the symplectic trivialization $\tau_{\gamma_{i}}$ of $\tilde{\xi}$ along $\gamma_{i}$. It is easy to see that the linearized return map $P_{\gamma_{i}}$ with respect to this trivialization is given by

$$
P_{\gamma_{i}}=\left(\begin{array}{ll}
\lambda^{k} & 0 \\
0 & \lambda^{-k}
\end{array}\right)
$$

Since the eigenvalues of $P_{\gamma_{i}}$ are positive real numbers different from $1, \gamma_{i}$ is a positive hyperbolic orbit. In addition, $P_{\gamma_{i}^{s}}=P_{\gamma_{i}}^{s}$. Therefore, the eigenvalues of $P_{\gamma_{i}^{s}}$ are different from 1 . Hence, $\gamma_{i}^{s}$ is a nondegenerate orbit for $s \in \mathbb{N}$ and $i=1, \ldots, n$. 
We now observe that the linearized Reeb flow around $\gamma_{i}$ (with respect to $\tau_{\gamma_{i}}$ ) rotates the eigenspaces of $P_{\gamma_{i}}$ by angle $-2 \pi$. Hence, we get

$$
\mu_{\tau_{\gamma_{i}}}\left(\gamma_{i}^{s}\right)=-2 s
$$

for $s \in \mathbb{N}$ and $i=1, \ldots, n$.

Now let the symplectic trivialization of $\xi_{\tilde{M}}$ along $[-N, N] \times\{p\}$ be given by the framing

$$
\left(\cos \left(\theta_{\delta, k, N}(t)\right) \partial_{x}+\sin \left(\theta_{\delta, k, N}(t)\right) \partial_{y},-\sin \left(\theta_{\delta, k, N}(t)\right) \partial_{x}+\cos \left(\theta_{\delta, k, N}(t)\right) \partial_{y}\right),
$$

where $\theta_{\delta, k, N}(t)=\pi(1-\delta k)(t+N) /(N k)$ and $t \in[-N, N]$. Note that $\left.R_{-k} \circ \varphi_{X_{H}}\right|_{V}$ is a rotation through $2 \pi(-1 / k+\delta)$, where $R_{-k}$ is a $-2 \pi / k$-rotation about $p$ and $\delta$ is a small positive irrational number. It is easy to see that with respect to this framing $P_{\gamma}$ is a rotation by $2 \pi(-1 / k+\delta)$. Hence, since $\delta$ is irrational, we see that $\gamma$ is an elliptic orbit and $\gamma^{t}$ is nondegenerate for $t \in \mathbb{N}$. Let

$$
N_{\delta}:=\max \{m \in \mathbb{N} \mid m \delta<1 / k\} .
$$

Note that we get

$$
\mu_{\tau_{\gamma}}\left(\gamma^{t}\right)=-2 m+1
$$

where ( $m-1) k<t \leq m k$ and $t \leq N_{\delta}$. Formulas (3D.2) and (3D.3) and the fact that $\delta$ is irrational imply that the parity of $\mu_{\tau_{\gamma_{i}}}\left(\gamma_{i}^{s}\right)$ is independent of $s$ for given $i$ and the parity of $\mu_{\tau_{\gamma}}\left(\gamma^{t}\right)$ is independent of $t$. Hence, we conclude that the $\gamma_{i}^{s}$ and $\gamma^{t}$ are good Reeb orbits for $i=1, \ldots, n$ and $s, t \in \mathbb{N}$.

It is not difficult to see that the symplectic trivialization $\tau_{\gamma^{k}}$ (induced from $\tau_{\gamma}$ ) can be extended to the $\tau_{\gamma_{i}}$ (are consistent in terms of Section 2B) along the surfaces obtained from $\left(\varphi_{X_{H}}^{(-N-t) / 2 N}\left(\gamma_{p, p_{i}}\right)\right)_{i=1}^{n k}$ by gluing them with $\varphi_{-k}$ and gives rise to the global symplectic trivialization that we call $\tau$.

\section{Calculation}

In this section, we calculate the sutured version of cylindrical contact homology of the sutured contact solid torus that we have constructed in Section 3.

Remark 4.1. There are no contractible Reeb orbits. Hence, from Theorem 2.7, Remark 2.11, and the fact that $\pi_{1}\left(S^{1} \times D^{2} ; \mathbb{Z}\right) \simeq H_{1}\left(S^{1} \times D^{2} ; \mathbb{Z}\right) \simeq \mathbb{Z}$ it follows that for all $h \in H_{1}\left(S^{1} \times D^{2} ; \mathbb{Z}\right), H C_{*}^{\mathrm{cyl}, h}\left(S^{1} \times D^{2}, \tilde{\Gamma}, \tilde{\alpha}_{\delta}, J\right)$ is defined, i.e., $\partial^{2}=0$, and is independent of contact form $\tilde{\alpha}_{\delta}$ for the given contact structure $\tilde{\xi}$ and the almost complex structure $J$.

For simplicity, assume that $l=1$. The calculation for $l>1$ can be made in the completely analogous way. 
Lemma 3.14 implies that all Reeb orbits are good and

$$
\left|\gamma_{i}^{s}\right|=-2 s-1, \quad\left|\gamma^{t}\right|=-2 m,
$$

where $m-1<t / k \leq m$ and $i=1, \ldots, n, s \leq N_{\delta} / k, t \leq N_{\delta}$. Hence, we get

(4A.5)

$$
C_{m}^{h}\left(\tilde{\alpha_{\delta}}, J\right)= \begin{cases}\mathbb{Q}\left\langle\gamma^{h}\right\rangle & \text { for } h>0 \text { and } m=2\lfloor h(-1 / k+\delta)\rfloor, \\ \mathbb{Q}\left\langle\gamma_{1}^{h / k}, \ldots, \gamma_{n}^{h / k}\right\rangle & \text { for } k \mid h>0 \text { and } m=-2 h / k-1, \\ 0, & \text { otherwise, }\end{cases}
$$

for $h \leq N_{\delta}$.

Now, since by Lemma $3.13 \mathscr{A}\left(\gamma^{k}\right)>\mathscr{A}\left(\gamma_{i}\right)$ for $i=1, \ldots, n$, we can use Fact 2.6 and Remark 2.11 and conclude that $\partial\left(\gamma_{i}^{s}\right)=0$ for $i=1, \ldots, n$ and $s>0$. Then, we prove that $\partial\left(\gamma^{t}\right)=0$ for $k \nmid t \leq N_{\delta}$. Since $\left[\gamma_{i}\right]=k[\gamma]$ in $H_{1}\left(S^{1} \times D^{2} ; \mathbb{Z}\right) \cong \mathbb{Z}$, the cylindrical contact homology differential at $\gamma^{t}$ counts only cylinders with negative end at $\gamma^{t}$. Then, similarly to the previous case, Fact 2.6 and Remark 2.11 imply that $\partial\left(\gamma^{t}\right)=0$ for $k \nmid t \leq N_{\delta}$.

We now consider the case when $k \mid t$ and will show that $\partial\left(\gamma^{t}\right) \neq 0$ for $k \mid t \leq N_{\delta}$. Is this situation, by arguing in the same way as in the case when $k \nmid t$, we get that $\partial\left(\gamma^{t}\right)$ counts only cylinders with negative end at $\gamma_{i}^{t / k}$.

Now we note that

$$
\operatorname{ind}(u)=\left|\gamma^{t}\right|-\left|\gamma_{i}^{t / k}\right|
$$

for any pseudoholomorphic curve $u$ in the moduli space $\mu^{J}\left(\gamma^{t} ; \gamma_{i}^{t / k}\right)$, where $k \mid t \leq N_{\delta}$ and $J$ is an almost complex structure tailored to $\left(\left(\mathbb{R} \times S^{1} \times D^{2}\right)^{*}, \tilde{\alpha}_{\delta}^{*}\right)$. The index formula can be written in this way, since $H_{2}\left(S^{1} \times D^{2} ; \mathbb{Z}\right)=0$ and hence $<c_{1}(\xi), A>=0$ for all $A \in H_{2}\left(S^{1} \times D^{2}, \mathbb{Z}\right)$. We now use (4A.4) and get

$$
\left|\gamma^{t}\right|-\left|\gamma_{i}^{t / k}\right|=-2 m-(-2 t / k-1)=-2(m-t / k)+1,
$$

and $m=t / k$ for $i=1, \ldots, n ; t \leq N_{\delta}$. Hence, we can rewrite (4A.6) as

$$
\operatorname{ind}(u)=\left|\gamma^{t}\right|-\left|\gamma_{i}^{t / k}\right|=-2(t / k-t / k)+1=1
$$

for $i=1, \ldots, n$ and $t \leq N_{\delta}$. Therefore, Theorem 2.8 and Remark 2.11 imply that for every $u \in M\left(\gamma^{t}, \gamma_{i}^{t / k}\right)$ the linearization of the Cauchy-Riemann operator is surjective at $u$; here $k \mid t \leq N_{\delta}, J$ is any almost complex structure tailored to $\left(\left(S^{1} \times D^{2}\right)^{*}, \tilde{\alpha}_{\delta}^{*}\right)$ and $i=1, \ldots, n$.

Let $\left(S^{1} \times D^{2}, \Gamma_{\text {long }}, U\left(\Gamma_{\text {long }}\right), \alpha_{\delta}^{\text {long }}\right)$ be a sutured contact solid torus obtained from $([-1,1] \times D, \Gamma, U(\Gamma), \alpha)$ by using $\varphi_{X_{H}}$ as a gluing map. Recall that we get $\left(S^{1} \times D^{2}, \tilde{\Gamma}, U(\tilde{\Gamma}), \tilde{\alpha}_{\delta}\right)$ from $([-1,1] \times D, \Gamma, U(\Gamma), \alpha)$ by using $\varphi_{-k}=R_{-k} \circ \varphi_{X_{H}}$ as a gluing map. We now note that $\left(S^{1} \times D^{2}, \Gamma_{\text {long }}, U\left(\Gamma_{\text {long }}\right), \alpha_{\delta}^{\text {long }}\right)$ is a universally tight sutured contact solid torus with $2 n k$ parallel longitudinal sutures, $k>1$, and 
such that when one cuts it along the meridian disk the sutures on the disk are boundary-parallel. This follows from the gluing/classification result for universally tight contact structures on a sutured solid torus; see Section 2 in [Honda 2002] (more precisely, Corollary 2.3, Theorem 2.5 and Corollary 2.6). The cylindrical contact homology of this sutured contact manifold is computed in [Golovko 2011] and is given by

$$
H C^{\mathrm{cyl}, h}\left(S^{1} \times D^{2}, \Gamma_{\text {long }}, \xi_{\text {long }}\right) \simeq \begin{cases}\mathbb{Q}^{n k-1} & \text { for } h \geq 1, \\ 0, & \text { otherwise }\end{cases}
$$

Here $\xi_{\text {long }}=\operatorname{ker} \alpha_{\text {long }}$.

Note that $\left(S^{1} \times D^{2}, \Gamma_{\text {long }}, U\left(\Gamma_{\text {long }}\right), \alpha_{\delta}^{\text {long }}\right)$ has $n k$ hyperbolic orbits

$$
\gamma_{1}^{\text {long }}, \ldots, \gamma_{n k}^{\text {long }}
$$

and one elliptic orbit $\gamma^{\text {long }}$. Here the $\gamma_{i}^{\text {long }}$ correspond to the equally spaced saddle points of $H$ and $\gamma^{\text {long }}$ corresponds to the critical point of $H$ at the center of $D\left(r_{\text {sing }}\right)$. In addition, observe that

$$
\left[\gamma_{i}^{\text {long }}\right]=\left[\gamma^{\text {long }}\right]=1 \in H_{1}\left(S^{1} \times D^{2} ; \mathbb{Z}\right) .
$$

Finally, note that from Lemma 3.13 and from the construction of

$$
\gamma^{\text {long }} \text { and } \gamma_{1}^{\text {long }}, \ldots, \gamma_{n k}^{\text {long }}
$$

it follows that

$$
\mathscr{A}\left(\gamma^{\text {long }}\right)>\mathscr{A}\left(\gamma_{i}^{\text {long }}\right), \quad \mathscr{A}\left(\gamma_{i}^{\text {long }}\right)=\mathscr{A}\left(\gamma_{j}^{\text {long }}\right)
$$

for $i, j=1, \ldots, n k$. Hence, Theorem 2.7, Remark 2.11 together with Fact 2.6, and (4A.8), (4A.9) and (4A.10) imply that $\partial\left(\gamma^{\text {long }}\right)^{s} \neq 0$ for $s>0$; otherwise we arrive at a contradiction with (4A.8) (since $\partial\left(\gamma^{\text {long }}\right)^{s}=0$ implies that the exponent of $\mathbb{Q}$ in (4A.8) must be $n k+1)$. In addition, observe that $<\partial\left(\gamma^{\text {long }}\right)^{s},\left(\gamma_{i}^{\text {long }}\right)^{s}>\neq 0$ for some $i$ and all $s>0$.

We now take an almost complex structure $J^{\text {long }}$ tailored to $\left(\left(S^{1} \times D^{2}\right)^{*},\left(\alpha_{\delta}^{\text {long }}\right)^{*}\right)$ such that as a map $\xi^{\text {long }} \rightarrow \xi^{\text {long }}$ it is obtained from some fixed $J^{\text {cyl }}: \xi \rightarrow \xi$ which is defined on $([-1,1] \times D, \Gamma, U(\Gamma), \alpha)$ and satisfies the following properties:

(1) $\left(J^{\mathrm{cyl}}\right)^{2}=-I, d \alpha\left(J^{\mathrm{cyl}} \cdot, J^{\mathrm{cyl}} \cdot\right)=d \alpha(\cdot, \cdot), d \alpha\left(\cdot, J^{\mathrm{cyl}} \cdot\right)>0$;

(2) $\left.J^{\mathrm{cyl}}\right|_{\{1\} \times D}=\varphi_{X_{H}}^{*}\left(\left.J^{\mathrm{cyl}}\right|_{\{-1\} \times D}\right)$ and $J^{\mathrm{cyl}}$ is $\frac{2 \pi}{n k}$-symmetric, i.e., it is invariant under $\frac{2 \pi}{n k}$-rotation with respect to the center of $D$.

Here $\xi^{\text {long }}=\operatorname{ker} \alpha_{\delta}^{\text {long }}$ and $\xi=\operatorname{ker} \alpha$. By saying that $J^{\text {long }}$ is obtained from $J^{\text {cyl }}$ we simply mean that the gluing procedure with $\varphi_{X_{H}}$ applied to $([-1,1] \times D, \Gamma, U(\Gamma), \alpha)$ transforms $J^{\text {cyl }}$ to $J^{\text {long }}$. Since $\xi$ is $\frac{2 \pi}{n k}$-symmetric on $([-1,1] \times D, \Gamma, U(\Gamma), \alpha)$, we 
claim that $J^{\mathrm{cyl}}$, which satisfies Properties (1) and (2), exists and that Property (2) is not a serious restriction on $J^{\text {cyl }}$. The symmetry of $\xi$ follows from the symmetry of $\beta$ and $X_{H}$, and from the construction of $\alpha$. From the symmetry of $J^{\text {long }}$ it follows that $<\partial\left(\gamma^{\text {long }}\right)^{s},\left(\gamma_{i}^{\text {long }}\right)^{s}>\neq 0$ for all $i=1, \ldots, n k$ and $s>0$.

Now we take $\tilde{J}$ on $\left(S^{1} \times D^{2}, \tilde{\Gamma}, U(\tilde{\Gamma}), \tilde{\alpha}_{\delta}\right)$, which is obtained from the same $J^{\text {cyl }}$ defined on $([-1,1] \times D, \Gamma, U(\Gamma), \alpha)$ by applying the gluing procedure with

$$
\varphi_{-k}=R_{-k} \circ \varphi_{X_{H}} \quad \text { to }([-1,1] \times D, \Gamma, U(\Gamma), \alpha),
$$

and possibly modify it near the boundary of $\left(S^{1} \times D^{2}, \tilde{\Gamma}, U(\tilde{\Gamma}), \tilde{\alpha}_{\delta}\right)$ (far from the Reeb orbits) so that it becomes tailored to $\left(\left(S^{1} \times D^{2}\right)^{*},\left(\tilde{\alpha}_{\delta}\right)^{*}\right)$. Observe that we can assume that $J^{\text {long }}=\tilde{J}$. From the symmetry of $J^{\text {cyl }}$ and the form of the gluing maps for $\left(S^{1} \times D^{2}, \tilde{\Gamma}, U(\tilde{\Gamma}), \tilde{\alpha}_{\delta}\right)$ and $\left(S^{1} \times D^{2}, \Gamma_{\text {long }}, U\left(\Gamma_{\text {long }}\right), \alpha_{\delta}^{\text {long }}\right)$ it follows that every $J^{\text {long }}$-holomorphic curve $u$ which contributes to $<\partial\left(\gamma^{\text {long }}\right)^{k s},\left(\gamma_{i}^{\text {long }}\right)^{k s}>\neq 0$ can be modified to a $\tilde{J}$-holomorphic curve $\tilde{u}$ from $\gamma^{k s}$ to $\gamma_{i}^{s}$ by modifying (composing) it with the rotation about the center of a meridian disk, and hence $<\partial \gamma^{k s}, \gamma_{i}^{s}>\neq 0$.

This choice of almost complex structures is possible since Theorem 2.8 and Remark 2.11 imply that we do not need to require almost complex structures to be generic. Finally, it follows from (4A.5) that

$$
H C_{m}^{\mathrm{cyl}, h}\left(S^{1} \times D^{2}, \tilde{\Gamma}, \tilde{\alpha}_{\delta}\right) \simeq \begin{cases}\mathbb{Q} & \text { for } h>0 \text { and } m=2\lfloor h(-1 / k+\delta)\rfloor, \\ \mathbb{Q}^{n-1} & \text { for } k \mid h>0 \text { and } m=-2 h / k-1, \\ 0, & \text { otherwise. }\end{cases}
$$

for $h \leq N_{\delta}$.

We now note that $\tilde{\xi}=\operatorname{ker} \tilde{\alpha}_{\delta}$ is independent of $\delta$. This follows from the gluing/classification result for universally tight contact structures on a sutured solid torus; see [Honda 2002, Corollary 2.3, Theorem 2.5 and Corollary 2.6]. Hence, from Theorem 2.7 and Remark 2.11 it follows that

$$
H C^{\mathrm{cyl}, h}\left(S^{1} \times D^{2}, \tilde{\Gamma}, \tilde{\xi}\right)=H C^{\mathrm{cyl}, h}\left(S^{1} \times D^{2}, \tilde{\Gamma}, \tilde{\alpha}_{\delta}\right)
$$

for all $h$ and hence for $h \leq N_{\delta}$, where $\delta$ is a small positive irrational number,

$$
\begin{aligned}
H C^{\mathrm{cyl}, h}\left(S^{1} \times D^{2}, \tilde{\Gamma}, \tilde{\xi}\right) & :=\bigoplus_{m} H C_{m}^{\mathrm{cyl}, h}\left(S^{1} \times D^{2}, \tilde{\Gamma}, \tilde{\xi}\right), \\
H C^{\mathrm{cyl}, h}\left(S^{1} \times D^{2}, \tilde{\Gamma}, \tilde{\alpha}_{\delta}\right) & :=\bigoplus_{m} H C_{m}^{\mathrm{cyl}, h}\left(S^{1} \times D^{2}, \tilde{\Gamma}, \tilde{\alpha}_{\delta}\right) .
\end{aligned}
$$

Now observe that $N_{\delta} \rightarrow \infty$ when $\delta \rightarrow 0$. In addition, we note that for fixed $n, k$ and two small positive irrational numbers $\delta_{1} \neq \delta_{2}$, the sets of closed orbits of $R_{\tilde{\alpha}_{\delta_{1}}}$ and $R_{\tilde{\alpha}_{\delta_{2}}}$ are the same, and the corresponding orbits with the same first homology class $h \leq \min \left\{N_{\delta_{1}}, N_{\delta_{2}}\right\}$ have the same Conley-Zehnder gradings in the corresponding 
complexes. Therefore, for every $0<h \in \mathbb{Z}=H_{1}\left(S^{1} \times D^{2} ; \mathbb{Z}\right)$, there exists $\delta$ such that

$$
\begin{aligned}
H C_{m}^{\mathrm{cyl}, h}\left(S^{1} \times D^{2}, \tilde{\Gamma}, \tilde{\xi}\right)=H C_{m}^{\mathrm{cyl}, h}\left(S^{1} \times D^{2}, \tilde{\Gamma}, \tilde{\alpha}_{\delta}\right) \\
\simeq \begin{cases}\mathbb{Q} & \text { for } h>0 \text { and } m=2\lfloor h(-1 / k+\delta)\rfloor, \\
\mathbb{Q}^{n-1} & \text { for } k \mid h>0 \text { and } m=-2 h / k-1, \\
0, & \text { otherwise. }\end{cases}
\end{aligned}
$$

for $h \leq N_{\delta}$ and hence

(4A.11) $H C_{m}^{\mathrm{cyl}, h}\left(S^{1} \times D^{2}, \tilde{\Gamma}, \tilde{\xi}\right) \simeq \begin{cases}\mathbb{Q} & \text { for } h>0 \text { and } m=2\left\lfloor-h / k+\delta_{k}\right\rfloor, \\ \mathbb{Q}^{n-1} & \text { for } k \mid h>0 \text { and } m=-2 h / k-1, \\ 0, & \text { otherwise, }\end{cases}$

where $0<\delta_{k} \ll 1 / k$. Finally, (4A.11) implies that

$$
H C^{\mathrm{cyl}, h}\left(S^{1} \times D^{2}, \Gamma, \xi\right) \simeq \begin{cases}\mathbb{Q} & \text { for } k \nmid h>0, \\ \mathbb{Q}^{n-1} & \text { for } k \mid h>0, \\ 0, & \text { otherwise. }\end{cases}
$$

This completes the proof of Theorem 1.1 when $l=1$.

For $l>1$, one can use the same observations as in the case when $l=1$ and show that the only nonzero part of the cylindrical contact homology differential is given by $<\partial \gamma^{t}, \gamma_{i}^{t / k}>\neq 0$ for $k \mid t \leq N_{\delta}$. This will lead to (4A.12) for all $l$ such that $(k, l)=1, k>l>0$.

Remark 4.2. Theorem 1.3 from [Golovko 2011] and Theorem 1.1 provide the formula for the sutured version of cylindrical contact homology of $\left(S^{1} \times D^{2}, \Gamma, \xi\right)$, where $\Gamma$ consists of $2 n$ parallel sutures of arbitrary slope, $\xi$ is a universally tight contact structure and such that if one cuts along the meridian disk, the sutures on the disk are $\partial$-parallel. In particular, this gives a complete calculation of the cylindrical contact homology of $\left(S^{1} \times D^{2}, \Gamma, \xi\right)$, where $\Gamma$ consists of 2 parallel sutures of arbitrary slope and $\xi$ is a universally tight contact structure (observe that in this situation there are only two isomorphic (but not isotopic) universally tight contact structures; see Section 2 in [Honda 2002]). These are not all the universally tight contact structures on the solid torus, but all of them can be obtained from the $\# \Gamma=2$ case by successively applying the folding operation.

\section{Acknowledgements}

The author is deeply grateful to Ko Honda for his guidance, help and support. He also thanks Dmytro Chebotarov, Oliver Fabert, Paolo Ghiggini, Jian He, Michael Hutchings and Mark McLean for helpful suggestions and interest in his work. In 
addition, the author is extremely grateful to Andrew Cotton-Clay for his critical comments on the first version of the paper. Also, the author is grateful to the referee of an earlier version of this paper for many valuable comments and suggestions. Finally, the author thanks the Mathematical Sciences Research Institute and the organizers of the "Symplectic and Contact Geometry and Topology" program for their hospitality.

\section{References}

[Bourgeois 2009] F. Bourgeois, "A survey of contact homology”, pp. 45-72 in New perspectives and challenges in symplectic field theory (Stanford, CA, 2007), edited by M. Abreu et al., CRM Proc. Lecture Notes 49, Amer. Math. Soc., Providence, RI, 2009. MR 2011a:53175 Zbl 1189.53082

[Bourgeois et al. 2003] F. Bourgeois, Y. Eliashberg, H. Hofer, K. Wysocki, and E. Zehnder, "Compactness results in symplectic field theory", Geom. Topol. 7 (2003), 799-888. MR 2004m:53152 Zbl 1131.53312

[Colin and Honda 2005] V. Colin and K. Honda, "Constructions contrôlées de champs de Reeb et applications”, Geom. Topol. 9 (2005), 2193-2226. MR 2006m:53134 Zbl 1091.53057

[Colin et al. 2011] V. Colin, P. Ghiggini, K. Honda, and M. Hutchings, "Sutures and contact homology I”, Geom. Topol. 15:3 (2011), 1749-1842. MR 2851076 Zbl 1231.57026

[Cotton-Clay 2009] A. Cotton-Clay, "Symplectic Floer homology of area-preserving surface diffeomorphisms”, Geom. Topol. 13:5 (2009), 2619-2674. MR 2011a:53173 Zbl 1179.37077

[Eliashberg et al. 2000] Y. Eliashberg, A. Givental, and H. Hofer, "Introduction to symplectic field theory", pp. 560-673 in Visions in mathematics: GAFA 2000 special volume, II (Tel Aviv, 1999), edited by N. Alon et al., Birkhäuser, Basel, 2000. MR 2002e:53136 Zbl 0989.81114

[Gabai 1983] D. Gabai, "Foliations and the topology of 3-manifolds", J. Differential Geom. 18:3 (1983), 445-503. MR 86a:57009 Zbl 0533.57013

[Golovko 2011] R. Golovko, "The embedded contact homology of sutured solid tori", Algebr. Geom. Topol. 11:2 (2011), 1001-1031. MR 2012g:53186 Zbl 1233.57019

[Honda 2002] K. Honda, "Gluing tight contact structures”, Duke Math. J. 115:3 (2002), 435-478. MR 2003i:53125 Zbl 1026.53049

[Hutchings 2002] M. Hutchings, "An index inequality for embedded pseudoholomorphic curves in symplectizations", J. Eur. Math. Soc. 4:4 (2002), 313-361. MR 2004b:53148 Zbl 1017.58005

[Momin 2011] A. Momin, "Contact homology of orbit complements and implied existence", J. Mod. Dyn. 5:3 (2011), 409-472. Zbl 1248.37057

[Wendl 2010] C. Wendl, "Automatic transversality and orbifolds of punctured holomorphic curves in dimension four", Comment. Math. Helv. 85:2 (2010), 347-407. MR 2011g:32037 Zbl 1207.32021

Received January 21, 2014. Revised June 15, 2014.

ROMAN GOLOVKO

DÉPARTEMENT DE MATHÉMATIQUES

UNIVERSITÉ PARIS-SUD

BÂTIMENT 425

91405 ORSAY

FRANCE

roman.golovko@math.u-psud.fr 


\title{
PACIFIC JOURNAL OF MATHEMATICS
}

\author{
msp.org/pjm
}

Founded in 1951 by E. F. Beckenbach (1906-1982) and F. Wolf (1904-1989)

\section{EDITORS}

Don Blasius (Managing Editor)

Department of Mathematics

University of California

Los Angeles, CA 90095-1555

blasius@math.ucla.edu

\author{
Paul Balmer \\ Department of Mathematics \\ University of California \\ Los Angeles, CA 90095-1555 \\ balmer@math.ucla.edu \\ Robert Finn \\ Department of Mathematics \\ Stanford University \\ Stanford, CA 94305-2125 \\ finn@math.stanford.edu \\ Sorin Popa \\ Department of Mathematics \\ University of California \\ Los Angeles, CA 90095-1555 \\ popa@math.ucla.edu
}

\author{
Vyjayanthi Chari \\ Department of Mathematics \\ University of California \\ Riverside, CA 92521-0135 \\ chari@math.ucr.edu \\ Kefeng Liu \\ Department of Mathematics \\ University of California \\ Los Angeles, CA 90095-1555 \\ liu@math.ucla.edu \\ Jie Qing \\ Department of Mathematics \\ University of California \\ Santa Cruz, CA 95064 \\ qing@ cats.ucsc.edu
}

\section{PRODUCTION}

Silvio Levy, Scientific Editor, production@msp.org

\section{SUPPORTING INSTITUTIONS}

ACADEMIA SINICA, TAIPEI

CALIFORNIA INST. OF TECHNOLOGY

INST. DE MATEMÁTICA PURA E APLICADA

KEIO UNIVERSITY

MATH. SCIENCES RESEARCH INSTITUTE

NEW MEXICO STATE UNIV.

OREGON STATE UNIV.

\author{
STANFORD UNIVERSITY \\ UNIV. OF BRITISH COLUMBIA \\ UNIV. OF CALIFORNIA, BERKELEY \\ UNIV. OF CALIFORNIA, DAVIS \\ UNIV. OF CALIFORNIA, LOS ANGELES \\ UNIV. OF CALIFORNIA, RIVERSIDE \\ UNIV. OF CALIFORNIA, SAN DIEGO \\ UNIV. OF CALIF., SANTA BARBARA
}

\author{
Daryl Cooper \\ Department of Mathematics \\ University of California \\ Santa Barbara, CA 93106-3080 \\ cooper@math.ucsb.edu \\ Jiang-Hua Lu \\ Department of Mathematics \\ The University of Hong Kong \\ Pokfulam Rd., Hong Kong \\ jhlu@maths.hku.hk \\ Paul Yang \\ Department of Mathematics \\ Princeton University \\ Princeton NJ 08544-1000 \\ yang@math.princeton.edu
}

These supporting institutions contribute to the cost of publication of this Journal, but they are not owners or publishers and have no responsibility for its contents or policies.

See inside back cover or msp.org/pjm for submission instructions.

The subscription price for 2015 is US \$420/year for the electronic version, and \$570/year for print and electronic.

Subscriptions, requests for back issues and changes of subscribers address should be sent to Pacific Journal of Mathematics, P.O. Box 4163, Berkeley, CA 94704-0163, U.S.A. The Pacific Journal of Mathematics is indexed by Mathematical Reviews, Zentralblatt MATH, PASCAL CNRS Index, Referativnyi Zhurnal, Current Mathematical Publications and Web of Knowledge (Science Citation Index).

The Pacific Journal of Mathematics (ISSN 0030-8730) at the University of California, c/o Department of Mathematics, 798 Evans Hall \#3840, Berkeley, CA 94720-3840, is published twelve times a year. Periodical rate postage paid at Berkeley, CA 94704, and additional mailing offices. POSTMASTER: send address changes to Pacific Journal of Mathematics, P.O. Box 4163, Berkeley, CA 94704-0163.

PJM peer review and production are managed by EditFLOW ${ }^{\circledR}$ from Mathematical Sciences Publishers.

\section{PUBLISHED BY}

\section{mathematical sciences publishers \\ nonprofit scientific publishing}

http://msp.org/

(C) 2015 Mathematical Sciences Publishers 


\section{PACIFIC JOURNAL OF MATHEMATICS}

Volume $274 \quad$ No. $1 \quad$ March 2015

Unimodal sequences and "strange" functions: a family of quantum modular forms

KATHRIN BRINGMANN, AMANDA FOLSOM and ROBERT C.

RHOADES

Congruence primes for Ikeda lifts and the Ikeda ideal

JIM BROWN and RODNEY KEATON

Constant mean curvature, flux conservation, and symmetry

NiCK EDELEN and BRUCE SOLOMON

The cylindrical contact homology of universally tight sutured contact solid tori

ROMAN GOLOVKO

Uniform boundedness of $S$-units in arithmetic dynamics

Holly Krieger, Aaron LeVin, Zachary Scherr, ThOMAS

TUCKER, Yu YASUFuKu and MiCHAEL E. ZiEVE

A counterexample to the energy identity for sequences of $\alpha$-harmonic 107 maps

YUXIANG LI and YOUDE WANG

Theory of newforms of half-integral weight

Murugesan MANiCKAM, JABAN MEHER and BALAKRISHNAN RAMAKRISHNAN

Algebraic families of hyperelliptic curves violating the Hasse principle

NGUYEN NGOC DONG QUAN

$F$-zips with additional structure

RichaRd PINK, TORSTEN WEDHORN and PAUL ZiEgler

Mean values of $L$-functions over function fields 